\title{
Direct numerical simulation of 'short' laminar separation bubbles with turbulent reattachment $\dagger$
}

\author{
By M. ALAM AND N. D. SANDHAM \\ School of Engineering Sciences, Aeronautics and Astronautics, University of Southampton, \\ Southampton, SO17 1BJ, UK
}

(Received 26 November 1998 and in revised form 13 September 1999)

Direct numerical simulation of the incompressible Navier-Stokes equations is used to study flows where laminar boundary-layer separation is followed by turbulent reattachment forming a closed region known as a laminar separation bubble. In the simulations a laminar boundary layer is forced to separate by the action of a suction profile applied as the upper boundary condition. The separated shear layer undergoes transition via oblique modes and $\Lambda$-vortex-induced breakdown and reattaches as turbulent flow, slowly recovering to an equilibrium turbulent boundary layer. Compared with classical experiments the computed bubbles may be classified as 'short', as the external potential flow is only affected in the immediate vicinity of the bubble. Near reattachment budgets of turbulence kinetic energy are dominated by turbulence events away from the wall. Characteristics of near-wall turbulence only develop several bubble lengths downstream of reattachment. Comparisons are made with two-dimensional simulations which fail to capture many of the detailed features of the full three-dimensional simulations. Stability characteristics of mean flow profiles are computed in the separated flow region for a family of velocity profiles generated using simulation data. Absolute instability is shown to require reverse flows of the order of $15-20 \%$. The three-dimensional bubbles with turbulent reattachment have maximum reverse flows of less than $8 \%$ and it is concluded that for these bubbles the basic instability is convective in nature.

\section{Introduction}

When a laminar boundary layer over a solid surface encounters a strong enough adverse pressure gradient it separates from the surface. The separated shear layer will usually undergo rapid transition to turbulence and the resulting turbulent flow may reattach to the surface and form an attached turbulent boundary layer. Such a flow phenomenon is known as a laminar separation bubble. Laminar separation bubbles are encountered in practical flows around aerofoils and in some circumstances control the aerodynamic performance. Examples are the leading-edge bubbles on thin aerofoils and the bubbles that form in high-lift multi-element aerofoil configurations. At incidences below the stall the transition from laminar to turbulent flow is fixed by the bubble, while the ultimate stall of the configuration is fixed by the 'bursting' of the bubble, where the flow no longer reattaches to the surface, or only reattaches much further downstream. In such practical applications the Reynolds numbers based on boundary layer momentum thickness at separation are of the order of $10^{2}$ to $10^{3}$

$\dagger$ This article first appeared in volume 403, pp. 223-250 but there were printing errors in some of the figure lettering. This reprinting replaces that version and will be the one that is referenced. 


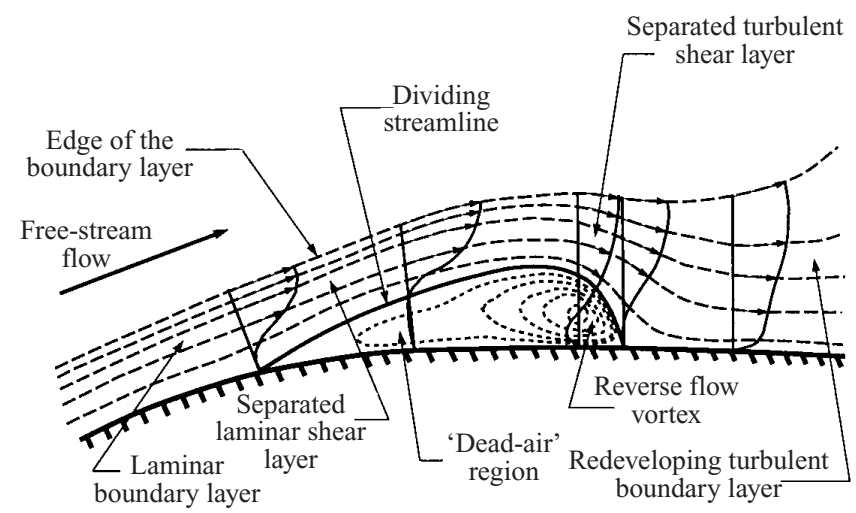

FiguRE 1. The mean flow structure of a laminar separation bubble (Horton 1968).

making the bubbles effectively low-Reynolds-number phenomena and hence suitable for the direct numerical simulation (DNS) approach adopted in this paper.

The first observations of laminar separation bubbles were published by Jones (1938). These observations and the interest in thin aerofoils for supersonic flight led to a series of experimental investigations into the fundamental structure and characteristics of laminar separation bubbles. Without reviewing all the work, we highlight some of the main conclusions that were arrived at towards the end of this experimental and theoretical phase of investigation in the 1960s. A more detailed review of the experiments was given by Young \& Horton (1966). The structure of a time-averaged bubble was given by Horton (1968) and reproduced on figure 1. Just downstream of the separation point the fluid close to the wall is virtually stationary and this region is referred to as the 'dead air' region. The separated shear layer, which is highly unstable, undergoes transition to turbulence and reattaches behind a vortical structure known as the 'reverse-flow vortex'. Bubbles such as this were classified (Owen \& Klanfer 1953) into two main types. 'Short' bubbles were found where the bubble length was of the order of $1 \%$ of the aerofoil chord and $10^{2} \delta_{s}^{*}$ to $10^{3} \delta_{s}^{*}$, where $\delta_{s}^{*}$ is the displacement thickness at separation, and 'long' bubbles with lengths of order $10^{4} \delta_{s}^{*}$ were also observed. In general short bubbles have only a small effect on the external potential flow whereas long bubbles completely alter the overall pressure distribution around an aerofoil. The original classification based on bubble length is application dependent and therefore not particularly helpful. However, it does make sense to distinguish bubbles that have only a local effect on the pressure distribution and we shall continue to use the term 'short bubble' to describe this case.

Thin aerofoils develop short laminar separation bubbles at moderate incidences and ultimately stall when the short bubble 'bursts' to form either a large bubble, with reattachment a long distance downstream, or without reattachment at all. Parameters governing bursting were identified by Gaster $(1963,1969)$, while Horton (1967) proposed a semi-empirical method for predicting the growth and bursting of bubbles. In this study we shall only touch briefly on bubble bursting when we discuss bubble stability characteristics, but we note that this is an important area of research where simulations should be able to play an important role.

The classical experiments of the 1950s and 1960s have recently begun to be supplemented with data from direct numerical simulations. In such simulations the governing equations are solved in full without modelling assumptions and a mixture of validation techniques is employed to ensure that the equations are accurately 
solved. When a simulation has been successfully validated databases are produced from which the flow structure and physics can be extracted. Complete simulations of laminar separation bubbles are more complex than simple channel and boundary layer flows and computers have only recently become large enough to tackle this problem, where a complete solution implies resolution of the reattached turbulent boundary layer in addition to the transition of the separated flow.

The first attempts to simulate laminar separation bubbles considered only the two-dimensional incompressible Navier-Stokes equations. Pauley, Moin \& Reynolds (1990), Ripley \& Pauley (1993), Lin \& Pauley (1996) and Wilson \& Pauley (1995) have at least partly reproduced experimental results for bubble structure. Small-scale structure has been included implicitly in the subgrid model for large-eddy simulations used by Wilson \& Pauley but the calculations were carried out only for two-dimensional large structures. Detailed comparisons of two-dimensional and fully three-dimensional simulations will be made later in this paper. Three-dimensional aspects of the transition process in laminar separation bubbles have been studied by Rist (1994), and Pauley (1994), although the simulations did not include full resolution of the turbulent reattachment region. Rist suggested a three-dimensional oblique mode breakdown rather than a secondary instability of finite-amplitude two-dimensional waves.

Only a few simulations with good resolution of the reattaching and developing turbulent boundary layer exist at present. Alam \& Sandham $(1997,1998)$ and Spalart \& Strelets (1997) have presented simulations of incompressible bubbles using spectral methods, while Wasistho (1998) has solved the compressible equations for bubbles in a flow at a free-stream Mach number $M_{\infty}=0.2$ with a high-order finite volume method. In the present work we present results from both two-dimensional and threedimensional simulations following numerical procedures outlined in $\S 2$. Transitional flow structures and the breakdown to turbulence are documented in $\S 3$. Budgets of Reynolds stresses are presented in $\S 4$ to study the properties of the flow downstream of the transition location and provide data relevant to modelling this region of the flow. A stability analysis of bubble velocity profiles is presented in $\S 5$, focusing on distinguishing whether the transition process in separation bubbles is triggered by a convective or absolute instability. This may have relevance to the bursting process. In $\S 6$ a detailed comparison of two-dimensional and three-dimensional bubble structure is presented, showing a limited applicability of the two-dimensional simulations.

\section{Direct numerical simulations}

\subsection{Problem definition and boundary conditions}

The adverse pressure gradient required for the formation of a laminar separation bubble can be reproduced in simulations by application of a suitable upper boundary condition. A method of suction through the upper boundary was used by Pauley et al. (1990, and in subsequent work), Spalart \& Strelets (1997) and Wasistho (1998), whereas Rist (1994) used a boundary condition for the free-stream velocity. Hildings (1997) has used two methods. He reported that bubble structure was sensitive to the precise specification of the upper boundary, but that it was possible to obtain good agreement with experiments using the suction approach. This fact had been reported earlier by Pauley et al. (1990) who made comparisons with Gaster's (1963, 1969) experiment. As described later, the differences between three-dimensional and two-dimensional simulations makes it difficult to judge the performance of boundary conditions based on agreement between two-dimensional simulations and experiments 


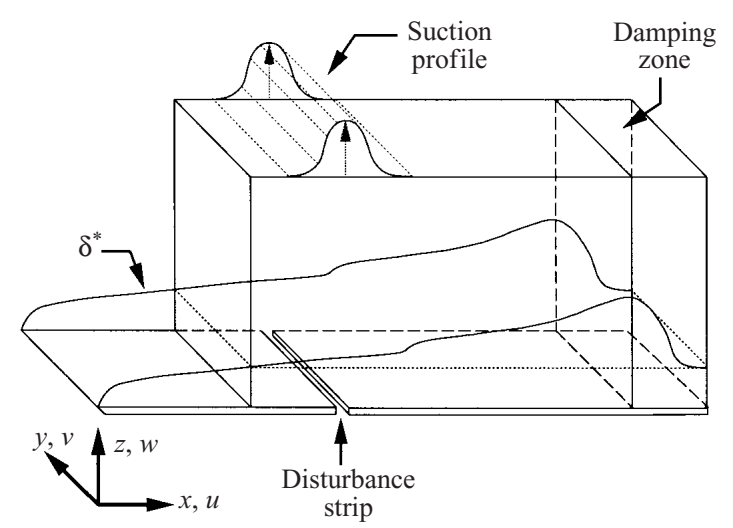

FIGURE 2. The computational domain.

which contain three-dimensional transition and turbulence. For reasons of simplicity of implementation we choose the suction method for the current work.

The computational box is shown on figure 2. The streamwise coordinate is $x$, spanwise $y$ and wall normal $z$. A Blasius velocity profile is prescribed at the inflow boundary and a no-slip condition is applied on the flat plate (lower boundary). At the upper boundary we apply a Gaussian suction (normal velocity) profile given by

$$
S(x)=a_{s} \exp \left[-b_{s}\left(x-c_{s}\right)^{2}\right],
$$

where three constants $a_{s}, b_{s}$ and $c_{s}$ control the size, shape and location of the suction profile. Dirichlet conditions $(u=1.0$ and $v=0.0)$ were applied to the other components.

In practical laminar separation bubbles transition occurs via amplification of naturally occurring background disturbances such as free-stream turbulence, sound, surface roughness or vibration. These are generally absent from a direct numerical simulation, except at the level of roundoff error, and so disturbances are applied to trigger the transition process. This is done by means of a disturbance strip applied on the lower boundary upstream of the separation point. Over a small streamwise extent, modulated by a Gaussian function, we apply disturbances to the wall-normal velocity that are sinusoidal in time and in the spanwise direction following a form

$$
w^{\prime}(x, y, t)=a_{f} \exp \left[-b_{f}\left(x-c_{f}\right)^{2}\right] \sin (\omega t) \sin (\beta y),
$$

where $a_{f}, b_{f}$ and $c_{f}$ are constants controlling the streamwise variation of the forcing, $\omega$ is the frequency and $\beta$ is the spanwise wavenumber. For two-dimensional simulations the term containing $y$ is omitted. The frequency $\omega$ is chosen to be in the range of unstable (or least damped) frequencies for both the Blasius boundary layer and the separated shear layer. Similarly the spanwise wavenumber $\beta$ is chosen to be in the unstable range. It was not felt necessary or appropriate to use eigenfunctions from linear stability analysis for the disturbances as in the separation bubble the laminar profiles are highly $x$-dependent so there is no 'correct' frequency or mode shape. The key is to trigger a transition that is typical in some sense of natural transition. A set of four simulations will be compared in later sections. Two of the simulations are two-dimensional, one of which is a forced case (2DF) and the other (2DS) is subjected to a much stronger suction in the absence of forcing. The simulation 3DF$\mathrm{A}$ is comparable to $2 \mathrm{DF}$ but with three-dimensional forcing. The simulation $3 \mathrm{DF}-\mathrm{B}$ has stronger suction strength and lower forcing amplitude compared to 3DF-A. 


\begin{tabular}{lcccccccccccc}
\hline Case & $a_{s}$ & $b_{s}$ & $c_{s}$ & $a_{f}$ & $b_{f}$ & $c_{f}$ & $\omega$ & $\beta$ & $a_{w}$ & $b_{w}$ & $c_{w}$ & $d_{w}$ \\
2DF & 0.15 & 0.02 & 25 & $30.08^{-3}$ & 0.125 & 10 & 0.15 & 0.0 & 3.77 & -0.056 & 181.5 & 187.5 \\
2DS & 0.21 & 0.02 & 25 & - & - & - & - & 0.0 & 3.77 & -0.056 & 181.5 & 187.5 \\
3DF-A & 0.15 & 0.02 & 25 & $30.08^{-3}$ & 0.125 & 10 & 0.15 & 0.41 & 3.77 & -0.056 & 181.5 & 187.5 \\
3DF-B & 0.20 & $9^{-3}$ & 29.25 & $15.04^{-3}$ & $73.96^{-3}$ & 10.83 & 0.15 & 0.41 & 3.77 & -0.033 & 175.5 & 182.5
\end{tabular}

TABLE 1. Numerical parameters of the suction and forcing profiles.

\begin{tabular}{|c|c|c|c|c|c|c|c|}
\hline Case & Type & Processor & \multicolumn{2}{|c|}{ Grid pts. } & $x_{L}\left(\delta_{i n}^{*}\right)$ & $y_{L}\left(\delta_{i n}^{*}\right)$ & $z_{L}\left(\delta_{i n}^{*}\right)$ \\
\hline $2 \mathrm{DF}$ & $2 \mathrm{D}$ & SGI R8000 & \multicolumn{2}{|c|}{$256 \times 4 \times 120$} & 200 & $\mathrm{~N} / \mathrm{A}$ & 10 \\
\hline $2 \mathrm{DS}$ & 2D & SGI R8000 & \multicolumn{2}{|c|}{$256 \times 4 \times 120$} & 200 & $\mathrm{~N} / \mathrm{A}$ & 10 \\
\hline 3DF-A & $3 \mathrm{D}$ & T3D (128 PE) & \multicolumn{2}{|c|}{$256 \times 128 \times 120$} & 200 & 30 & 10 \\
\hline $3 \mathrm{DF}-\mathrm{B}$ & $3 \mathrm{D}$ & T3E (64 PE) & \multicolumn{2}{|c|}{$384 \times 128 \times 160$} & 200 & 30 & 13 \\
\hline & & \multicolumn{6}{|c|}{ TABLE 2. Computational grid and box sizes. } \\
\hline Case & $R e_{\delta_{i n}^{*}}$ & $\%$ for & cing amp. & $l_{b} /$ & $\theta_{s}$ & $\delta_{i n}^{*}$ & $R e_{l t}$ \\
\hline $2 \mathrm{DF}$ & 500 & 230 & 3.0 & 49.3 & & 46 & 7845 \\
\hline 2DS & 700 & 315 & $0.46^{*}$ & 105. & & 45 & 25255 \\
\hline 3DF-A & 500 & 246 & 3.0 & 33.4 & & 49 & 6667 \\
\hline $3 \mathrm{DF}-\mathrm{B}$ & 500 & 335 & 1.5 & 42.3 & & 67 & 11837 \\
\hline
\end{tabular}

The basic parameters for the upper suction profile and lower disturbance strip are shown on table 1 . Numerical parameters and some key properties computed from the simulations are shown on tables 2 and 3 .

In order to use a fully spectral numerical method periodic boundary conditions are applied in the streamwise direction. The physical problem is not periodic, so we employ an artificial 'buffer' zone at the end of the computational domain to return the turbulent outflow to the Blasius laminar inflow profile. Such an approach was originally used by Spalart (e.g. Spalart \& Watmuff 1993) and has also been employed by Guo, Kleiser \& Adams (1994) for boundary layer transition problems and by Hildings (1997) for two-dimensional simulations of separation bubbles. The location of the buffer zone is fixed by a function

$$
f(x)= \begin{cases}a_{w} \exp \left[b_{w}\left\{\left(x-c_{w}\right)^{2}+\left(x-d_{w}\right)^{2}\right\}\right] & \text { for } x_{b}<x<L_{x} \\ 0 & \text { otherwise. }\end{cases}
$$

Constants controlling the buffer function are $a_{w}, b_{w}, c_{w}$ and $d_{w}$. Numerical values are shown on table 1 . The constant $x_{b}$ locates the start of the buffer region and is set close to 160 for all the simulations shown here. $L_{x}$ is the box length. Detailed implementation is described in the next section. Here we note that in the 'physical' computational domain the incompressible Navier-Stokes equations are solved in full. In the buffer zone a modified set of equations is solved with the criteria that the flow recovers as closely as possible to the specified Blasius inflow condition without numerical instabilities developing. The success of the buffer zone is judged by $a$ posteriori investigation of the flow at the inflow, which at some level will contain disturbances that have propagated through the buffer zone. Typically the maximum kinetic energy of these disturbances is one order of magnitude lower than the kinetic 
energy due to the disturbance strip. In this case the transition process in controlled by the specified disturbances while the disturbances that have leaked through the buffer zone serve to break symmetries faster than would otherwise happen.

\subsection{Numerical method}

The pseudo-spectral method used for this study is originally due to Kleiser \& Schumann (1980). Pressure and viscous terms are treated implicitly with the CrankNicolson method while the convective terms are treated explicitly. A small change relative to the original method is the use of a third-order Runge-Kutta method rather than Adams-Bashforth for the explicit part. In this subsection we outline the modifications to the method for the separation bubble simulations. More details of the Kleiser-Schumann method are given in Canuto et al. (1988). The new implementation of the method for massively parallel computers is described in Sandham \& Howard (1998).

The governing equations are written in a non-dimensional form using the incoming free-stream velocity $U_{\infty}$ and the displacement thickness of the incoming boundary layer $\delta_{i n}^{*}$ as reference quantities. The continuity equation is

$$
\nabla \boldsymbol{u}=0,
$$

where $\boldsymbol{u}$ is the velocity vector, and the momentum equations are

$$
\frac{\partial \boldsymbol{u}}{\partial t}=\boldsymbol{u} \times \boldsymbol{\omega}-\nabla q+\frac{1}{R e} \nabla^{2} \boldsymbol{u},
$$

where the Reynolds number is $R e=U_{\infty} \delta_{i n}^{*} / v, v$ being the kinematic viscosity. The convective terms are written in rotational form using the vorticity vector $\omega$, with $q$ a modified pressure $q=p+\boldsymbol{u} \cdot \boldsymbol{u} / 2$.

At each Runge-Kutta substep the following equation must be solved:

$$
\frac{\boldsymbol{u}^{n+1}-\boldsymbol{u}^{n}}{\Delta t}=a(\boldsymbol{u} \times \boldsymbol{\omega}+\boldsymbol{M})^{n}+b(\boldsymbol{u} \times \boldsymbol{\omega}+\boldsymbol{M})^{n-1}-\frac{\nabla q^{n+1}+\nabla q^{n}}{2}+\frac{\nabla^{2} \boldsymbol{u}^{n+1}+\nabla^{2} \boldsymbol{u}^{n}}{2 \operatorname{Re}},
$$

where $a$ and $b$ are constants for the Runge-Kutta method, superscripts $n-1, n$ and $n+1$ refer to successive substeps, and

$$
\boldsymbol{M}=\Delta \boldsymbol{u} f(x)
$$

is an extra term that applies only in the buffer zone where $f(x)$ is non-zero and $\Delta \boldsymbol{u}$ is the difference between the actual velocity vector and the required inflow. By this mechanism the outflow turbulent boundary layer is returned smoothly to the inflow condition, allowing the use of Fourier expansions in the streamwise coordinate direction. For solution, terms in (6) are expanded in Fourier series in the periodic directions $x$ and $y$, while Chebyshev expansions are used in the wall-normal direction $z$. Solution of coupled equations for $q$ and $w$ follows the method of Kleiser \& Schumann, using a Chebyshev tau method to solve a sequence of Helmholtz and Poisson equations. The convective terms are de-aliased using the $\frac{3}{2}$ rule, i.e. $\boldsymbol{u}$ and $\omega$ are expanded by $50 \%$ before transformation to real space where the nonlinear products are computed. Truncation of the additional modes occurs after back transformation to wave space, where the remaining operations are carried out. The de-aliasing operation is potentially a barrier to efficient parallelisation. A method for constructing an efficient code on massively parallel computers is given in Sandham \& Howard (1998). This approach is followed in the current work where simulations have been carried out on Cray T3D and T3E computers using up to 128 processors. The simulations 


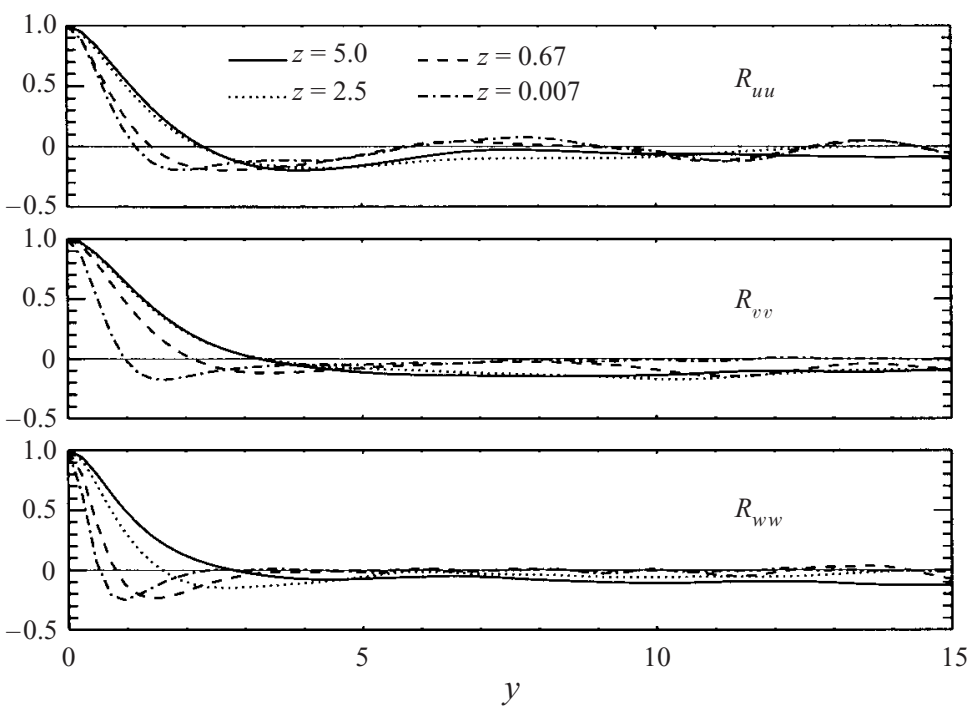

FIGURE 3. Spanwise two-point correlations $R_{u u}, R_{v v}$ and $R_{w w}$ at $x=110$ (turbulent part downstream of reattachment) for the wall-normal locations shown at the top.

3DF-A and 3DF-B used 20000 PE hours on T3D and 10500 PE hours on T3E respectively.

\subsection{Validations}

A variety of validations have been carried out for the results presented in this paper. More complete descriptions are given in Alam (1998). The basic numerical method and parallel code are the same as used for simulations of turbulent channel flow (Sandham \& Howard 1998; Howard \& Sandham 1997). These have been compared in detail with the reference channel flow simulations of Kim, Moin \& Moser (1987). Additionally this code was checked for the laminar channel flow solution and by comparison with results from linear stability analysis for the growth of small disturbances. For the spatial code with a buffer zone it was checked that the boundary-layer evolution without application of suction matched results from a separate laminar boundarylayer solver. Further validations were conducted for flows with separation bubbles present. To check the spanwise box size, two-point correlation data were accumulated. Figure 3 shows the correlations of velocities as a function of spanwise distance at a typical location in the turbulent part of the boundary layer downstream of reattachment.

The decay of the correlations to zero indicates that the box is large enough in the spanwise direction. The simulation results do depend on the location of the upper boundary simply because the effect of the suction profile changes. The box size in the normal direction was fixed so that the redeveloping boundary layer does not reach the upper boundary at the furthest streamwise location. The box length in the streamwise direction was set as large as possible to compute more of the boundary-layer relaxation.

A useful check of resolution is a comparison with other spectral simulations of turbulent wall-bounded flows. In table 4 we compare the grid spacings in terms of wall units with the turbulent boundary-layer simulation of Spalart (1988) and the 


$\begin{array}{ccccc}\text { Case } & \Delta x^{+} & \Delta y^{+} & \Delta z^{+} \text {at } z^{+}=9 & N\left(z^{+}<9\right) \\ \text { KMM } & 11.78 & 7.00 & 1.33 & 13 \\ \text { Spalart } & 20.00 & 6.70 & - & 10 \\ \text { 3DF-A } & 20.73 & 6.20 & 0.90 & 16 \\ \text { 3DF-B } & 14.26 & 6.30 & 0.87 & 17 \\ \text { TABLE 4. Comparison of resolution with other simulations. ( } N \text { is the number of grid points in the } \\ \text { near-wall region.) }\end{array}$

channel flow of Kim et al. (1987). The wall units are defined by

$$
\Delta x^{+}=\frac{\Delta x u_{\tau}}{v},
$$

where $\Delta x$ is the grid spacing and $u_{\tau}=\sqrt{\tau_{w} / \rho}, \tau_{w}$ being the shear stress at the wall. Resolution in the spanwise $y$-direction is evaluated in a similar manner, while in the wall-normal direction resolution is compared by tabulating the number of points for $z^{+}<9$. Results from the three-dimensional simulations 3DF-A and 3DF-B are evaluated at the skin friction peak, which is expected to be the most difficult region of the flow to resolve. It can be seen that the resolution is everywhere comparable to or better than that of Spalart.

A final, but very important, check is the computation of Reynolds stress budgets. These are described in more detail in $\S 4$. For validation purposes one must check that the left-hand side (convection terms) of the transport equations for Reynolds stresses is balanced by the right-hand side which contains production, destruction and transport terms. Experience with the current code is that under-resolution of the flow results in a significant imbalance in the equations. The budgets of all the Reynolds stresses demonstrated an acceptable balance, sufficient for a detailed discussion of turbulence statistics up to the third moments that appear in the transport terms of the equations.

\subsection{Main characteristics of the bubbles}

In this subsection an overview of results of three-dimensional simulations of laminar separation bubbles is presented. Comparisons with two-dimensional simulations are delayed until $\S 6$. One general point to make is the difficulty found in relating simulation results to boundary-layer properties. The suction zone that is used to force the bubbles has the effect of locally distorting the potential flow such that in the vicinity of the bubble there is no single value of the local free-stream velocity $U_{e}$ that can be used to evaluate boundary-layer properties such as displacement thickness. There is, however, a clear distinction between vortical fluid inside the boundary layer and irrotational flow outside it, which led Spalart \& Strelets (1997) to propose the use of a pseudo-velocity given by integration of the spanwise vorticity

$$
\bar{U}(x, z)=-\int_{0}^{z} \omega_{y} \mathrm{~d} z^{\prime} .
$$

This enables comparisons to be made with boundary-layer properties and the same approach is followed here. 


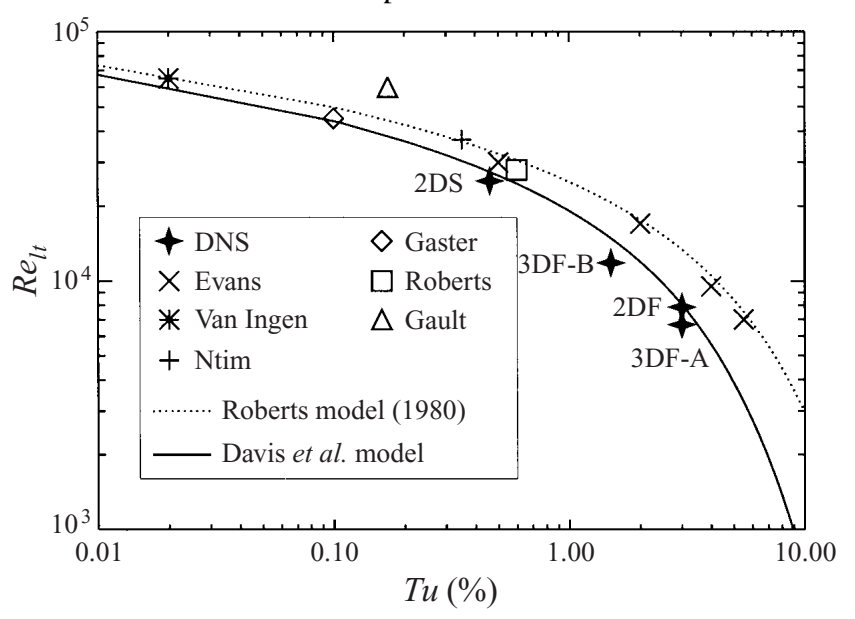

Figure 4. Variation of Reynolds number based on transition length with local turbulence level. Experimental results are taken from Davis et al. (1985).

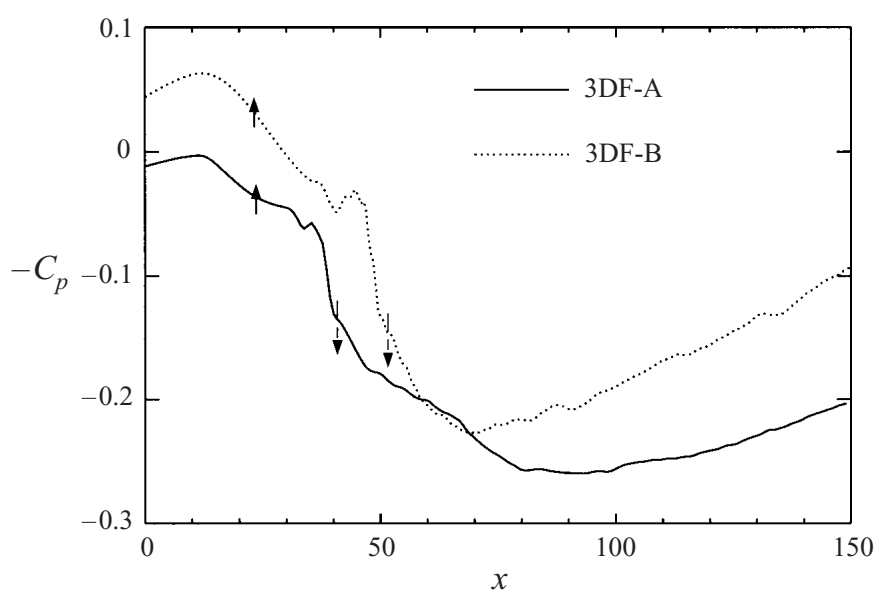

FIGURE 5. Pressure coefficient of the three-dimensional bubbles with turbulent reattachment.

\subsubsection{Mean flow properties}

Tables 2 and 3 show the grids and box lengths used for the simulations and key non-dimensional parameters derived from the results, using the pseudo-velocity (9) for boundary-layer quantities. Important quantities are the Reynolds number based on the boundary-layer momentum thickness at separation $\theta_{s}$ and the 'transition length' Reynolds number based on distance from separation to the point of transition. The transition point is taken here to be at the point of maximum negative $c_{f}$. Also shown is the ratio of bubble length $l_{b}$ to $\theta_{s}$ and the ratio of $\theta_{s}$ to inflow displacement thickness $\delta_{i n}^{*}$. A comparison with experiments is shown on figure 4, which plots the transition-length Reynolds number against turbulence intensity (the square root of the turbulence kinetic energy). For the simulations the turbulence intensity is taken from the amplitude of the forcing. It can be seen that the two simulations follow the trends of the experimental results and the models given by Davis, Carter \& Reshotko (1985) and Roberts (1980). Figures 5 and 6 show distributions of the coefficient of pressure $C_{p}$ and the skin friction $c_{f}=\tau_{w} /\left(\frac{1}{2} \rho U_{e}^{2}\right)$. The bubble separation and reattachment points are located by the zero crossings of the skin friction plots. In each case 


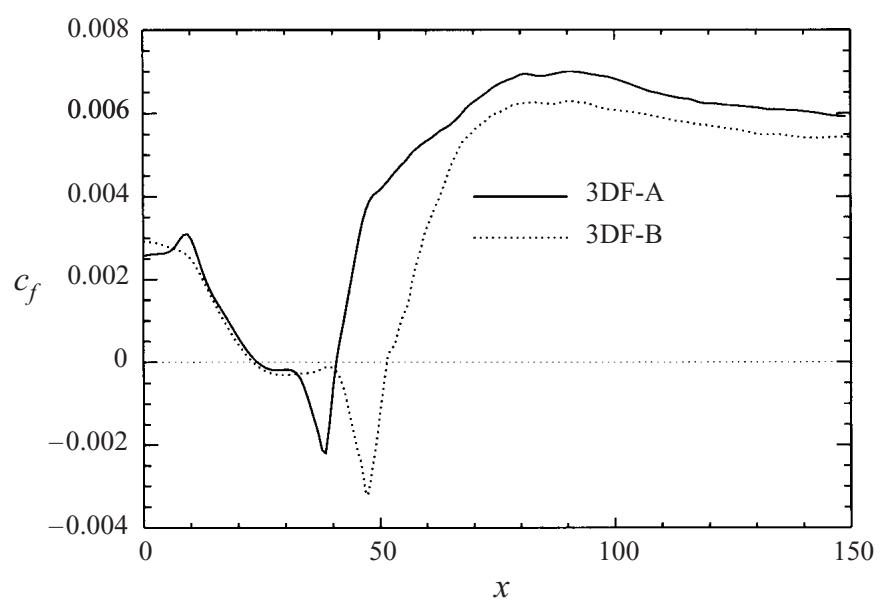

FIGURE 6. Skin friction variation of the three-dimensional bubbles.

the bubble is located in the region of strongest adverse pressure gradient and the reattachment occurs before the end of the adverse pressure gradient region. There is a departure from the expected distribution only in the vicinity of the bubble, and so it is concluded that the bubbles simulated are of the 'short' type when compared with the classical experiments. It can be seen that the stronger suction of case $3 \mathrm{DF}-\mathrm{B}$ results in a longer region of separated flow but with the same general behaviour. Some distance upstream and downstream of the bubble the pressure gradient is mildly favourable. A characteristic feature of each bubble is the flat skin friction distribution in the dead-air region, with a much larger negative skin friction occurring below the reverse-flow vortex.

The mean flow structure is illustrated by velocity contours on figure 7. One should note that with all such figures the coordinate normal to the surface has been stretched to better illustrate the structure. It should be kept in mind that the actual bubbles are very shallow flow phenomena. The shear-layer spreading in the reattachment region and the development of high velocity gradients near the wall in the developing turbulent boundary layer are clear. The maximum reverse flow is equal to $4 \%$ of the inflow $U_{\infty}$ for simulation 3DF-A and $8 \%$ for 3DF-B.

\subsubsection{Probability density functions}

A distorted view of laminar separation bubbles may result if one only considers the mean flow. The actual flow, especially in the reattachment region, is highly unsteady and the flow structure shown on figure 7 is never seen in instantaneous views. In this section we study the extent of the unsteadiness of the flow by considering probability density functions (PDFs) of the skin friction in different parts of the flow from simulation 3DF-A. The PDFs are accumulated by sorting the instantaneous skin friction at 100 different streamwise locations into any of 59 bins. The bin boundaries are given by

$$
c_{f_{i}}=c_{f_{\min }}+\frac{(i-1)}{59}\left(c_{f_{\max }}-c_{f_{\min }}\right) \quad \text { for } i=1,60
$$

with $c_{f_{\min }}=-0.01$ and $c_{f_{\max }}=0.017$. Checks were made that the range of $c_{f}$ was sufficient. Statistics were accumulated over two cycles of the imposed forcing at the locations shown on figure 7 , with results shown on figure 8 . The first plot, figure $8(a)$ is taken before separation and the flow is always moving forward. The 

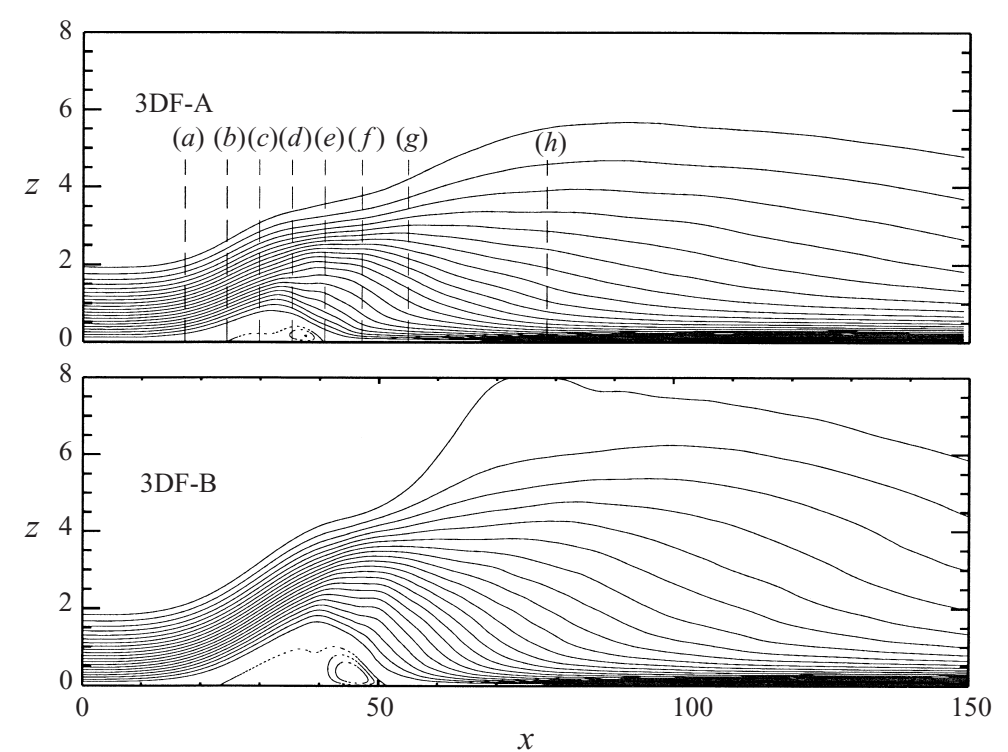

FIGURE 7. Streamwise velocity contours of the three-dimensional bubbles with turbulent reattachment. PDFs of skin friction have been calculated at locations $(a-h)$ (see figure 8). Maximum contour level is 0.88 and minimum contour level is -0.04 for both cases.

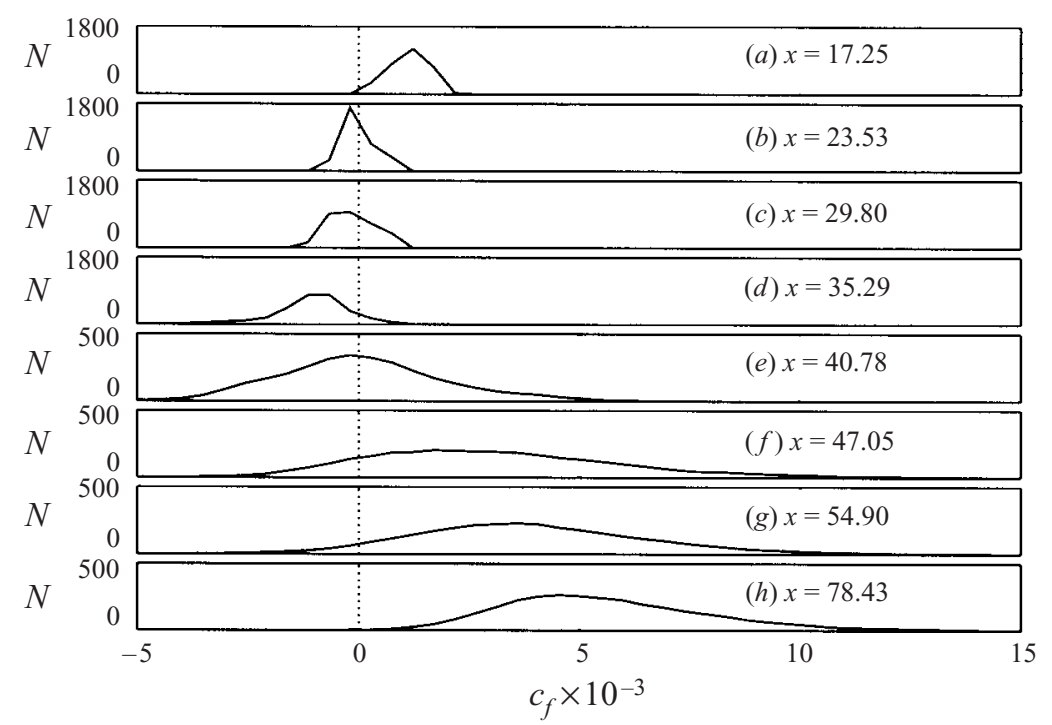

FIGURE 8. PDFs of skin friction along a bubble at locations defined on figure 7.

mean separation point corresponds to figure $8(b)$ and the mean reattachment to figure $8(e)$. In between one can note that there is no location where the flow is always reversed. Especially in the dead-air region there is a significant proportion of the time when the flow is moving forward. Even underneath the reverse-flow vortex the flow does not always have negative $c_{f}$. At reattachment the PDF is almost symmetric while downstream of reattachment significant reverse flow exists, even on figure $8(g)$ which is nearly one bubble length downstream of reattachment. As a summary figure 9 shows the percentage of time that the flow is forward and reversed as a function 


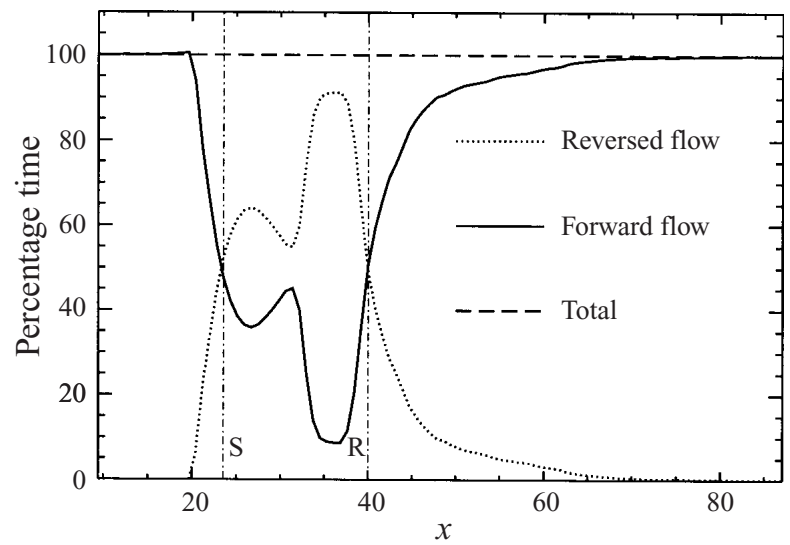

Figure 9. Composition of the mean flow in terms of forward/reversed flow.

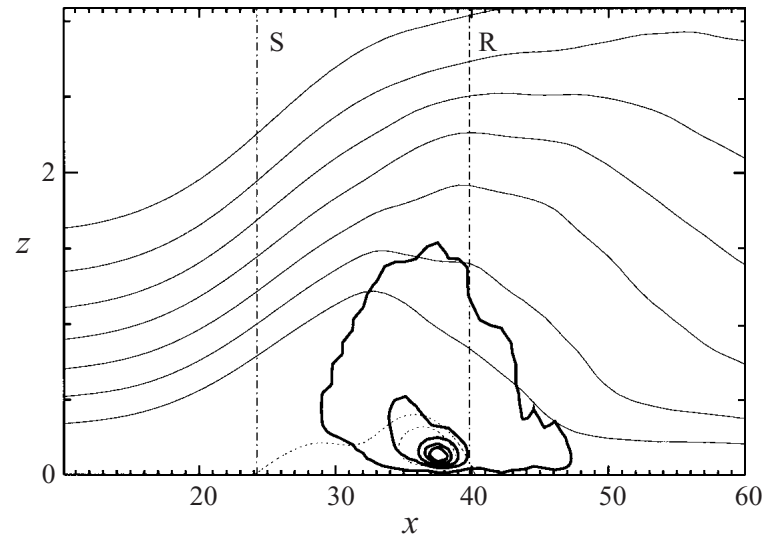

FIGURE 10. PDF of reverse flow in the range $5-10 \%$. Bold contour lines show the percentage time the flow is within $5-10 \%$ reverse flow. The levels of the lines are: outer, $0.01 \%$; second outer, $10 \%$; middle, $20 \%$; second inner, $30 \%$; inner, $35 \%$.

of streamwise location. The $50 \%$ points coincide with the mean separation and reattachment locations.

Another type of PDF can be found by accumulating the times when the reverse flow is in a certain specified range in the flow. This will be useful later when the stability characteristics of separation bubble profiles are considered. Figure 10 shows an example where contours are shown of the amount of time that the flow is actually reversed in the range of 5-10\% of $U_{e}$. These contours are shown in bold, with lighter contours of mean velocity serving to locate the bubble. It can be seen that there is only a small region near the mean reverse-flow vortex where reverse flows of this magnitude exist for even $30 \%$ of the time. In this region reverse flows of $0-5 \%$ are seen approximately $50 \%$ of the time, while reverse flows of $10-15 \%$ are seen only $3.5 \%$ of the time and reverse flows of above $15 \%$ are seen less than $1 \%$ of the time.

\section{Flow structures in the transitional and reattaching flow regions}

Flow structures in fully developed turbulence and in the late stages of breakdown to turbulence have already been studied for several canonical flows (e.g. Robinson 


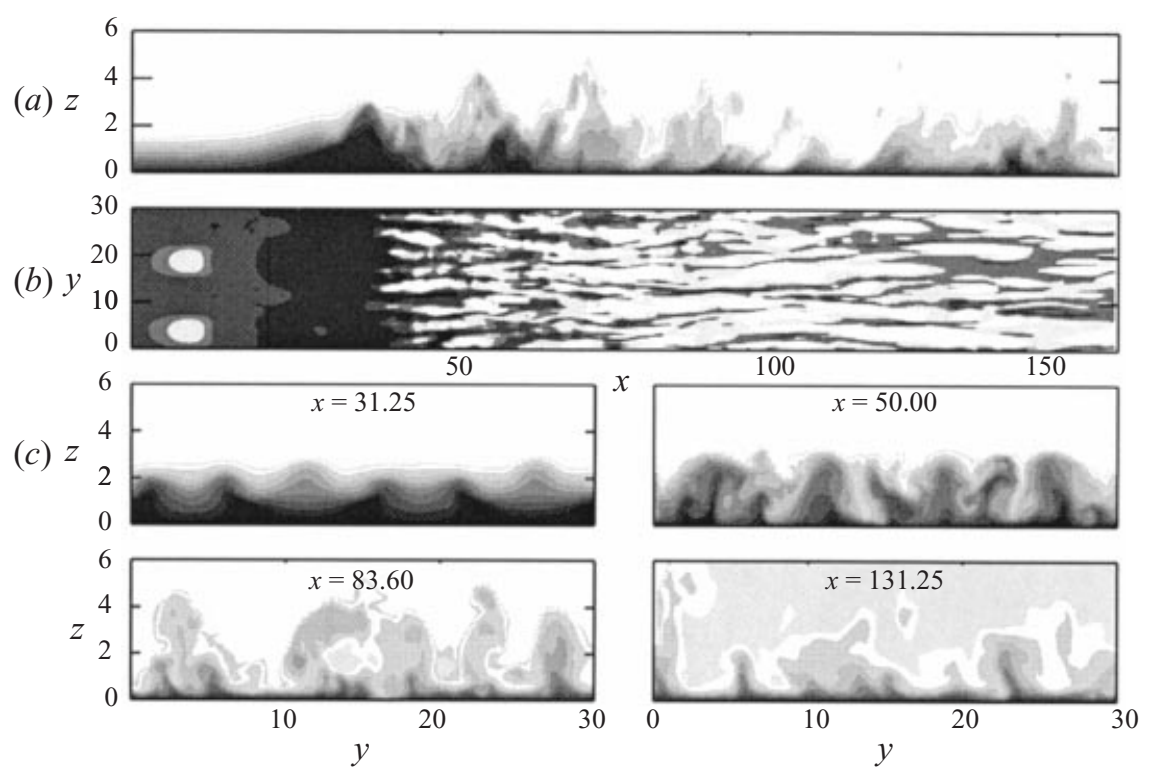

FIGURE 11. Instantaneous streamwise velocity contours of a bubble with turbulent reattachment (3DF-A) (maximum level is 0.8 , minimum level is -0.14 and the darkest colour shows the reversed flow). (a) $(x, z)$-plane, $(b)(x, y)$-plane, and (c) slices of $(y, z)$-plane.

1991; Sandham \& Kleiser 1992). In this subsection we consider briefly the nature of the breakdown to turbulence that occurs in the separated shear layer and the flow structures that appear in the emerging turbulent boundary layer downstream of reattachment.

Velocity contours illustrate some important features of the instantaneous flow. Figure 11 shows grey-scale contours of streamwise velocity $(a)$ in the side view $(x, z$ plane) at $y=30.0,(b)$ in the top view ( $x, y$ plane) at $z=0.06$ and $(c)$ in the end view $(y, z$ plane $)$ at $x=31.25,50.0,83.6$ and 131.25. The darkest colour shows where the flow is separated. The side view may be compared with the time-averaged plot, figure 7. A pocket of separated flow can be seen downstream of the mean reattachment at $x \approx 60$. The top view shows the development of low-speed streaks in the reattaching boundary layer. These characteristic structures of near-wall turbulent flow are clearly visible for $x>80$. The symmetry loss during transition can be observed in figure $11(\mathrm{c})$. The initial spanwise symmetry about $y=3 L_{y} / 8$ is slightly disturbed at $x=31.25$ and completely broken by $x=83.6$.

Vorticity contours are shown on figure 12(a) for the wall-normal component $\omega_{z}=$ $\partial u / \partial y-\partial v / \partial x$ and $(b)$ for the spanwise component $\omega_{y}=\partial w / \partial x-\partial u / \partial z$. The transition region is characterized by staggered large-scale $\Lambda$-vortices visible in the surface of $\omega_{z}$. These $\Lambda$-vortices pump fluid away from the wall. By a vorticity stretching process, very similar to that seen by Sandham \& Kleiser (1992) for K- and H-type transition in Poiseuille flow, a detached shear layer forms above these vortices, visible in the surface of $\omega_{y}$. The surface labelled $\mathrm{A}$ on figure $12(b)$ is formed by the $\Lambda$-vortex labelled A on figure 12(a). A similar connection exists for the surfaces labelled B on these figures. The $\Lambda$-vortex legs extend behind the mean separation due to the growth of fluctuations in the unstable adverse pressure gradient boundary layer upstream of separation.

As can be seen from the discussion of the previous paragraph, vorticity is evidence 

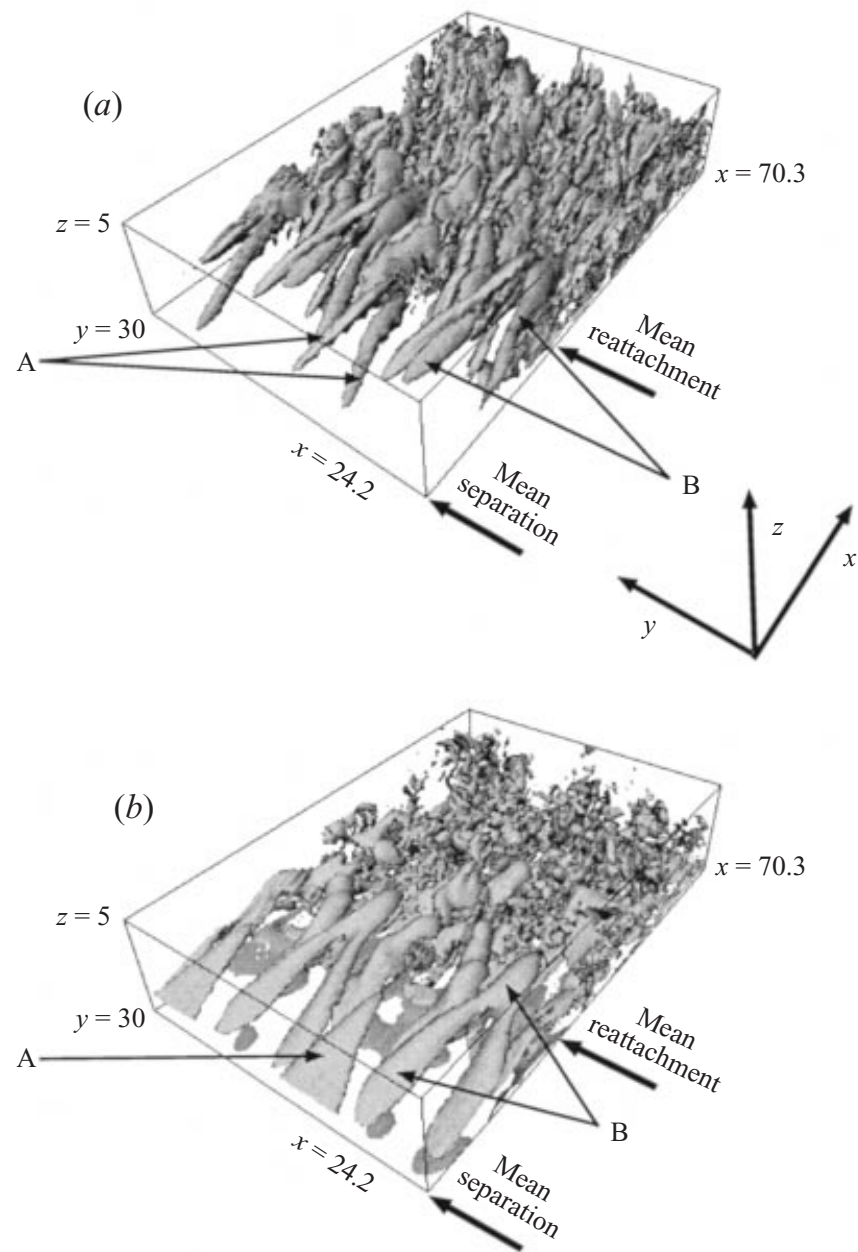

FIGURE 12. (a) Surface plot of wall-normal vorticity $\left(\omega_{z}=\mathrm{d} u / \mathrm{d} y-\mathrm{d} v / \mathrm{d} x\right)$. Identifiable structures: (A) $\Lambda$ structure located in the separated shear layer, (B) a distorted $\Lambda$ structure about to break up near the reattachment point (see figure 13 for more details for time sequence). (b) Surface plot of spanwise vorticity $\left(\omega_{y}=\mathrm{d} w / \mathrm{d} x-\mathrm{d} u / \mathrm{d} z\right)$. Various identifiable structures: (A) shear layer forming above the $\Lambda$-vortex structure labelled A in (a), (B) a $\Lambda$-shaped shear layer about to break up.

for either vortices or shear layers. To distinguish between them we need more precise methods to define vortices. Here, three different techniques have been used: (i) static pressure relative to local mean pressure, (ii) static pressure relative to the local mean wall pressure, and (iii) second invariant of the velocity gradient tensor $\left(\mathrm{II}=\left(\partial u_{i} / \partial x_{j}\right)\left(\partial u_{j} / \partial x_{i}\right)\right)$. Low pressure and negative second invariant define the location of a vortex. Subtraction of the wall pressure sometimes helps to locate a vortex that exists inside a larger region of pressure gradient. Using these methods we focus on the structures in the breakdown region that can be properly classified as vortices. Figure 13 shows a time series of views of the flow near reattachment using method (i). The series proceeds from left to right and top to bottom. To illustrate the mechanisms we consider the evolution of the structure labelled $\mathrm{B}$ in the figure. This structure can also be found on figure 12(a) where it first appeared as the stronger left side of an asymmetric $\Lambda$-vortex. The shear layers that form as this vortex moves 


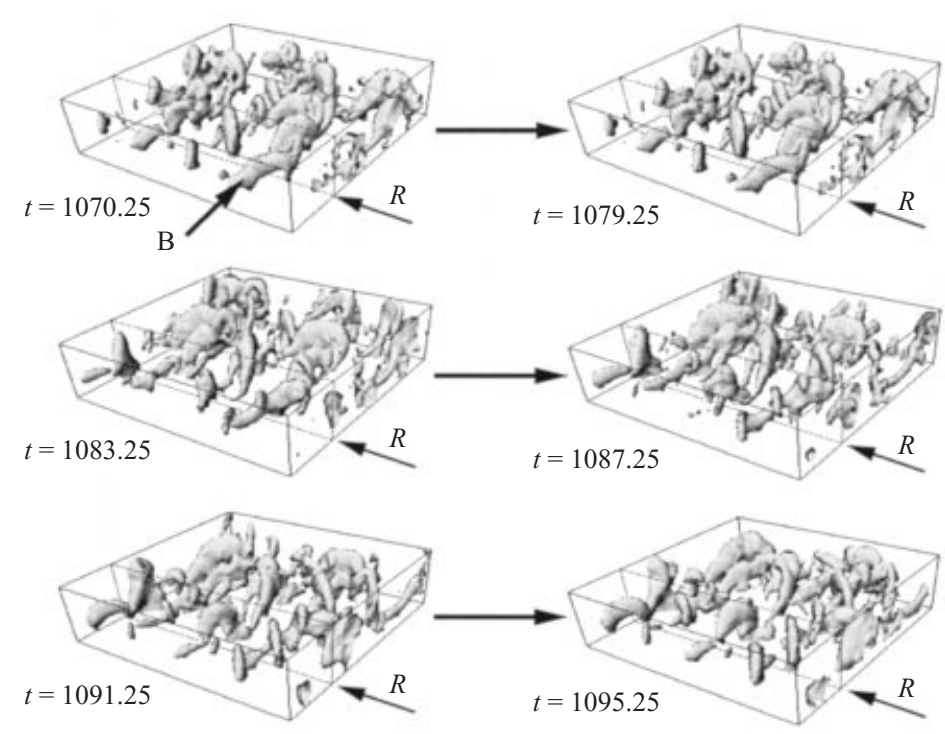

FIGURE 13. Time sequence surface plot of $p^{\prime}=-0.01524$ in the vicinity of mean reattachment (from $x=31.8$ to $x=62.5$ ). The structure labelled B corresponding to structure B in figure $12(a)$ can be traced through break up via secondary vortices perpendicular to the original.

Regions

Dead-air region \& shear layer

Reattachment region

Relaxing boundary layer
Characteristic features

(i) $\Lambda$-shaped structures visible by $u^{\prime}, v^{\prime}, w^{\prime}, \omega_{x}, \omega_{y}$ and $\omega_{z}$.

(ii) $\Lambda$ vortices visible by $p^{\prime}, p_{w}^{\prime}$ and $\mathrm{B}$.

(iii) Transient regions of reversed and forwardly moving flow.

(i) Hairpin vortices visible by $p^{\prime}$.

(ii) Transient regions of reversed flow.

(i) One/two-sided hairpin vortices visible by $p^{\prime}$.

(ii) Streaks (quasi-streamwise vortices) visible by $u$ and $u^{\prime}$.

(iii) Transient regions of reversed flow.

TABLE 5. Characteristic flow structures for different regions of the flow.

low-speed fluid away from the wall themselves roll up into vortices which can be seen at $t=1083.25$. The smaller vortices appear above the original vortex with a principal axis perpendicular to the original one. The structure thus formed is reminiscent of the model proposed by Theodorson (1955) of a horseshoe vortex superimposed on a horseshoe vortex. It is now known that such structures are not common in turbulent boundary layers (Robinson 1991). Nevertheless it is interesting that such a structure can be seen to exist as a transient solution of the Navier-Stokes equations. In the further evolution the original vortex decays and one is left with a series of three new vortices skewed in the opposite sense to the original $\Lambda$-vortex leg. This is an example of a cascade to smaller scales caused by vorticity stretching. A similar process can be seen occurring at other points in the flow.

Flow structures observed in different parts of the flow are summarized in table 5. In our simulations complete breakdown to turbulence happens near the reattachment position rather than in the separated flow region. More simulations are needed to 
confirm that reattachment follows the early breakdown stage of $\Lambda$-vortices rather than complete breakdown to turbulence.

\section{Turbulent boundary-layer relaxation}

\subsection{Turbulence structure and budgets}

The turbulence kinetic energy, Reynolds stress and pressure fluctuation fields are shown by contour plots on figure 14, taken from simulation 3DF-A. Upstream of separation the kinetic energy input from the disturbance strip is visible at $x=10$. This kinetic energy decays downstream until the highly unstable separated shear layer is encountered. Here the kinetic energy is amplified reaching a maximum half a bubble length downstream of reattachment. In this region the shear layer spreads rapidly away from the wall, while close to the wall steep gradients in kinetic energy and $\left\langle u^{\prime} u^{\prime}\right\rangle$ develop.

Transport equations for turbulence kinetic energy (TKE) and Reynolds stress provide more information relevant to modelling flows. Budgets from all the Reynolds stresses for the separation bubble simulation 3DF-A are given in Alam (1998). Here we focus on the turbulence kinetic energy equation which can be written

$$
\frac{\partial k}{\partial t}+\left\langle u_{i}\right\rangle \frac{\partial k}{\partial x_{i}}=P-\varepsilon-\frac{\partial}{\partial x_{i}}\left(J_{i}^{u}+J_{i}^{p}+J_{i}^{v}\right),
$$

where

$$
\begin{aligned}
P & =-\left\langle u_{i}^{\prime} u_{j}^{\prime}\right\rangle \frac{\partial\left\langle u_{i}\right\rangle}{\partial x_{j}} & & \text { (Production), } \\
\varepsilon & =\frac{1}{\operatorname{Re}}\left\langle\frac{\partial u_{i}^{\prime}}{\partial x_{j}}\left[\frac{\partial u_{i}^{\prime}}{\partial x_{j}}+\frac{\partial u_{j}^{\prime}}{\partial x_{i}}\right]\right\rangle & & \text { (Dissipation), } \\
J_{i}^{u} & =\left\langle u_{i}^{\prime} u_{j}^{\prime} u_{j}^{\prime}\right\rangle / 2 & & \text { (Triple moment tran } \\
J_{i}^{p} & =\left\langle p^{\prime} u_{i}^{\prime}\right\rangle & & \text { (Pressure transport), } \\
J_{i}^{v} & =-\frac{1}{\operatorname{Re}}\left[\frac{\partial k}{\partial x_{i}}+\frac{\partial\left\langle u_{i}^{\prime} u_{j}^{\prime}\right\rangle}{\partial x_{j}}\right] & & \text { (Viscous transport). }
\end{aligned}
$$

Complete budgets are shown on figure 15 at three streamwise locations: $(a) x=35$, which is inside the bubble near the reverse-flow vortex, $(b) x=62$, about one bubble length downstream of reattachment, and $(c) x=156$, which is about six bubble lengths downstream of reattachment and close to the end of the useful part of the simulation. The normal coordinate for all of these plots is taken as $z_{t}^{+}$which is $z^{+}$using $u_{\tau}$ in the developed turbulent boundary layer at $x=156$. This allows the spreading of the turbulence in the normal direction to be observed, while retaining a useful scale for the developing turbulent boundary layer. The differences between the structures of the budget are striking. At the first location all the significant nonzero terms are in the separated shear layer. Here the key balance is between on the one hand convection, which is negative (primarily due to the $\bar{u} \partial k / \partial x$ term, since $k$ increases rapidly with $x$ during transition), and on the other hand production and triple moment transport which tend to increase $k$. At this first location dissipation plays only a minor role. At the second location, after reattachment, the dominant turbulence activity is in the range $40<z_{t}^{+}<180$ and consists mainly of a balance of production, dissipation, triple-moment transport, pressure transport and convection. The last three all change sign in the range $70<z^{+}<85$. These results may be 


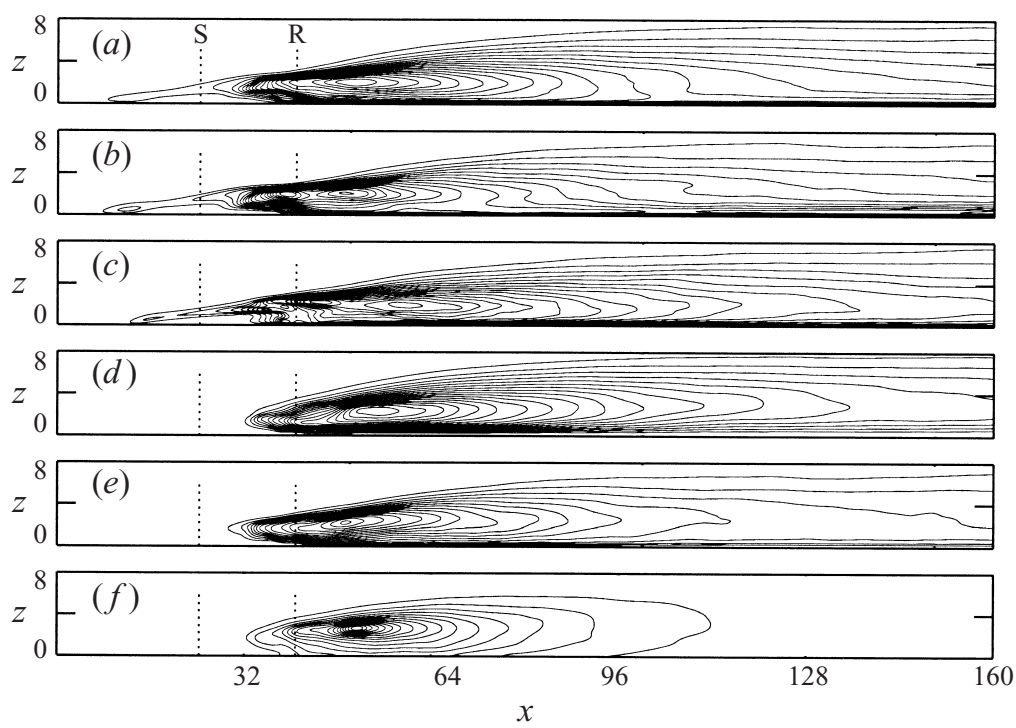

FIGURE 14. Contours of fluctuation statistics of a three-dimensional bubble (3DF-A): $(a) k,(b)$ $\left\langle u^{\prime} u^{\prime}\right\rangle,(c)\left\langle v^{\prime} v^{\prime}\right\rangle,(d)\left\langle w^{\prime} w^{\prime}\right\rangle,(e)\left\langle u^{\prime} w^{\prime}\right\rangle,(f)\left\langle p^{\prime} p^{\prime}\right\rangle$. Maximum contour levels are 0.0248, 0.0282, 0.0131, $0.0119,6.31 \times 10^{-5}$ and 0.0013 respectively.

compared with Rogers \& Moser (1994) who simulated self-similar turbulent mixing layers. Similar sign changes occurred in their simulations at values of the similarity coordinate $0.9<\xi<1.8$. Relative magnitudes of all the terms are also close to the curves from Rogers $\&$ Moser, and thus the region $z^{+}>60$ can be well represented as the upper half of a mixing layer flow. Close to the wall a new peak in production emerges and the dissipation is high and balanced by the viscous transport term. This is indicative of emerging near-wall turbulent flow. By the third location the shear layer activity is much diminished and the dominant balance is of production and dissipation, except for close to the wall where triple moment and viscous transport terms are important. This balance is the same as that seen in turbulent channel and boundary-layer flows.

As figure $15(b)$ at $x=62$ contains characteristics of both a free shear layer (for $\left.z_{t}^{+}>60\right)$ and a newly formed wall boundary layer $\left(z_{t}^{+}<20\right)$, we present full Reynolds stress budgets for this point in the flow on figure 16. As with the kinetic energy equation, transport terms are divided into pressure transport, turbulence transport (due to the triple moment of velocity fluctuations), and a viscous transport term involving the Reynolds number (Sandham \& Howard 1998). The pressure strain can be seen to act as a redistribution of $\left\langle u^{\prime} u^{\prime}\right\rangle$ into $\left\langle v^{\prime} v^{\prime}\right\rangle$ and $\left\langle w^{\prime} w^{\prime}\right\rangle$. Pressure transport is significant for the $\left\langle w^{\prime} w^{\prime}\right\rangle$ and $\left\langle u^{\prime} w^{\prime}\right\rangle$ components.

\subsection{Boundary-layer relaxation}

The boundary layer downstream of reattachment is initially very different from an equilibrium turbulent boundary layer. Velocity profiles at several downstream locations are shown on figure 17 in the usual semi-logarithmic format using wall variables. A slow relaxation of the profiles towards equilibrium is observed. It takes until the furthest downstream location before the profiles approach the accepted logarithmic law of the wall. This location corresponds to seven bubble lengths downstream of reattachment or $\left(x-x_{R}\right) / \delta_{R}=24.43$, where $x_{R}$ is the reattachment 


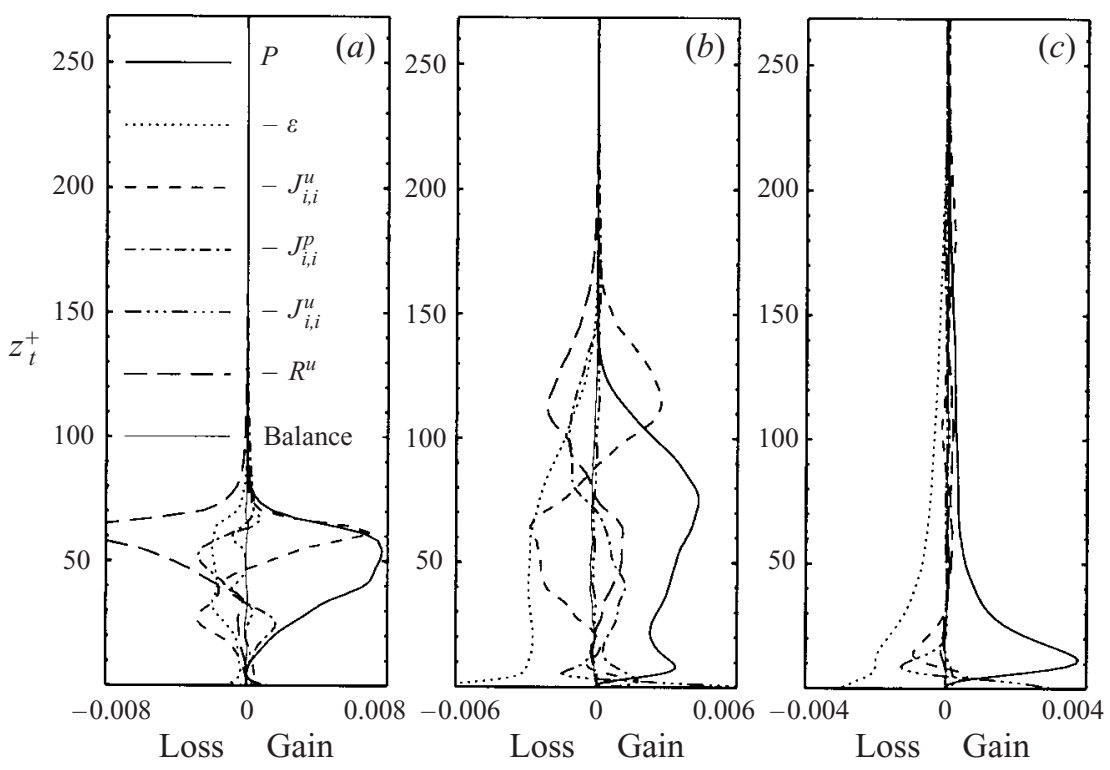

FIGURE 15. Balance of TKE at three different locations $((a-c) x=35,62$ and 156 respectively).
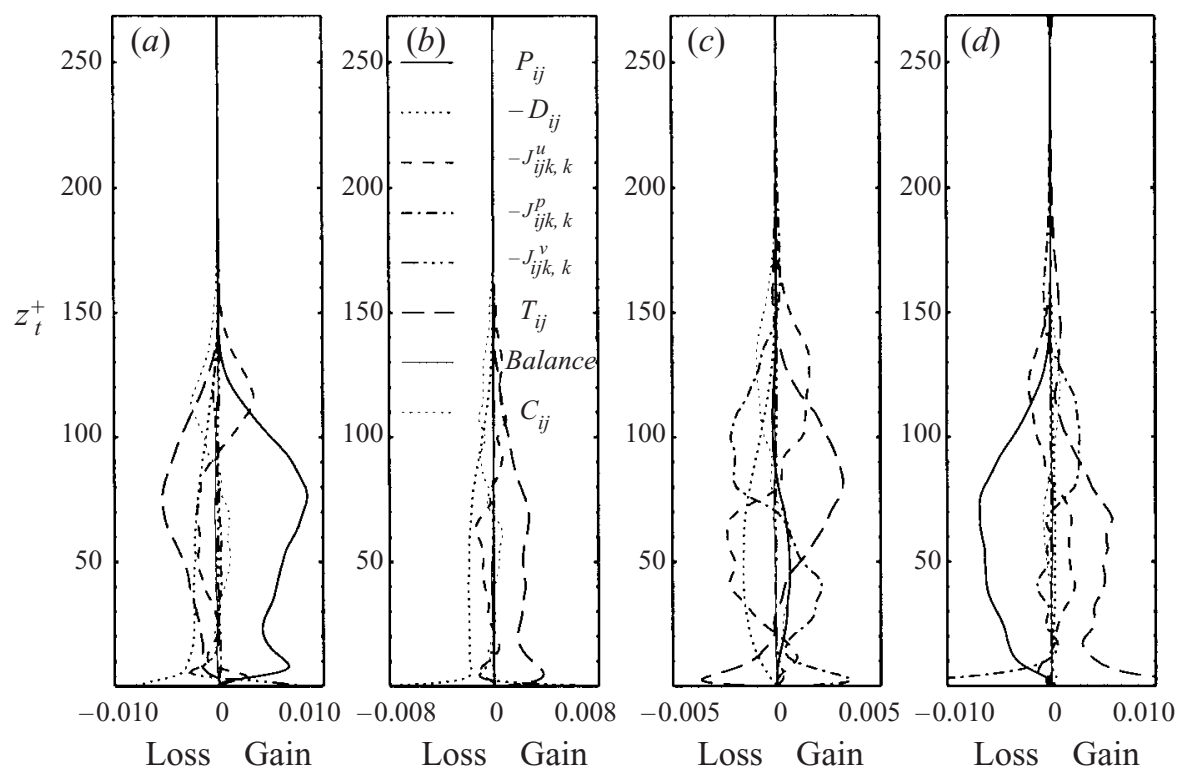

Figure 16. Balance of Reynolds stresses (at $x=62)$ of a three-dimensional bubble (3DF-A). (a) $\left\langle u^{\prime} u^{\prime}\right\rangle,(b)\left\langle v^{\prime} v^{\prime}\right\rangle,(c)\left\langle w^{\prime} w^{\prime}\right\rangle$ and $(d)\left\langle u^{\prime} w^{\prime}\right\rangle$.

location and $\delta_{R}$ is the $99.5 \%$ boundary-layer thickness at reattachment. Up to that point the profiles all lie below the log law. Similar behaviour was found in the experiments of Castro \& Epik (1996) for the boundary layer behind a separation bubble on a flat plate with a blunt leading edge, and in the compressible simulation of a laminar separation bubble by Wasistho (1998). It has also been observed in experiments and simulations of the backward-facing step flow (e.g. Bradshaw \& Wong 1972; Le, Moin \& Kim 1997) suggesting that the phenomenon is common to all cases 


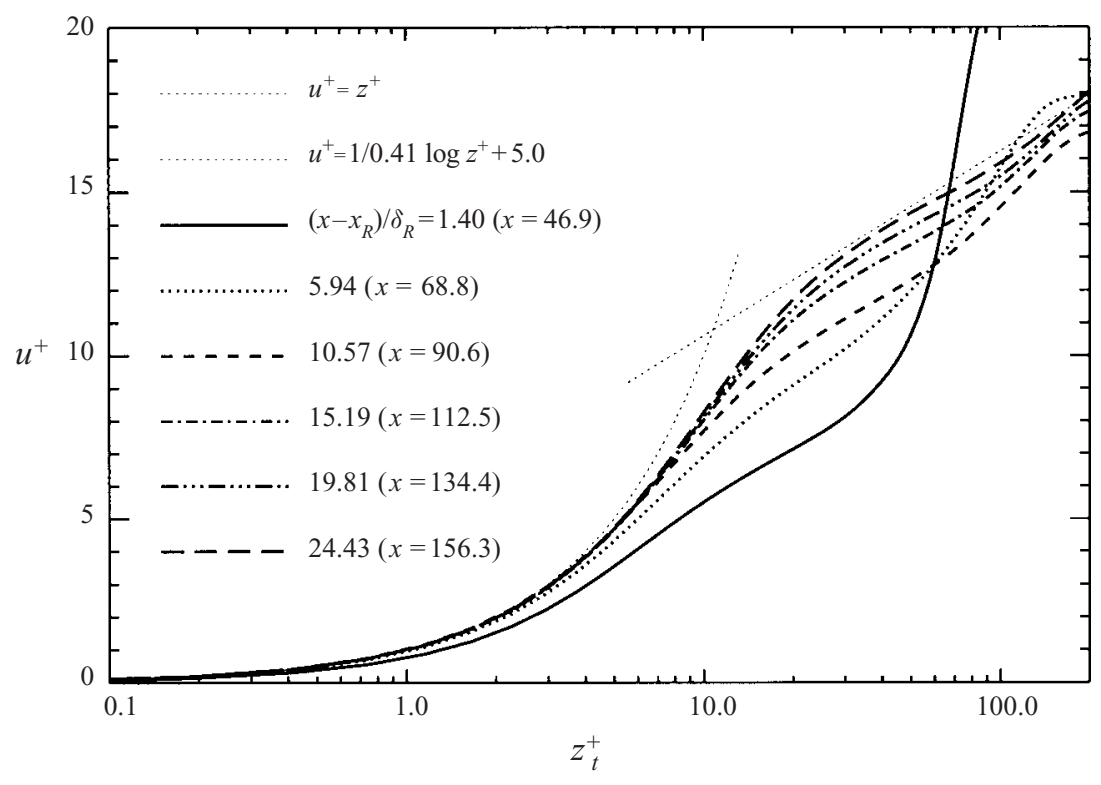

FIGURE 17. Streamwise velocity profiles of the relaxing boundary layer.

of turbulent reattachment. In this region the 'Clauser plot' method of determining skin friction is clearly inapplicable. Various explanations of the phenomena have been offered. Most recenty Le et al. attribute the effect to adverse pressure gradient and low Reynolds number effects. Indeed adverse pressure gradients do seem to displace the law of the wall downwards as shown in the simulations of Spalart \& Watmuff (1993). However low Reynolds numbers do not give downward shifts in the log law (Spalart 1988). In our current application much of the redevelopment occurs in a region of small favourable pressure gradient (see figure 5) and we prefer an explanation based on the increased $(\partial\langle u\rangle / \partial z)_{w}$, and hence $u_{\tau}$, due to high levels of cross-stream momentum transfer in the immediate aftermath of transition where the turbulence is highly energetic. Wasistho (1997) makes a useful analogy with the effect of a rough wall, and hence increased turbulence activity, which also leads to a downward displacement of the log law.

The Clauser parameter $G=(H-1) / H \sqrt{2 / c_{f}}$ gives some idea of the approach to equilibrium. Figure 18 shows a plot of $G$ against $\left(x-x_{R}\right) / \delta_{R}$ for the two threedimensional simulations. The expected equilibrium value is 6.8 for a flat-plate zero pressure gradient boundary layer. This value may not be appropriate to the present work due to the slight favourable pressure gradient and the low Reynolds numbers. However there is evidence from Castro \& Epik (1996) that recovery lengths of more than $75 \delta_{R}$ are required to reach equilibrium, which would require much longer computational boxes than those considered here. One aspect of the recovery that can be computed from the simulations is the distance from reattachment to the peak of skin friction, $l_{r}$, which occurs in the boundary layer downstream of reattachment. This is shown on figure 19 plotted as a function of bubble length, with both axes normalized by momentum thickness at separation. Comparisons are made with data from Wasistho (1998) and Spalart \& Strelets (1997). The solid line is a correlation to the data

$$
\frac{l_{r}}{l_{b}}=\frac{1}{2.4-69\left(\theta_{s} / l_{b}\right)} \text {. }
$$




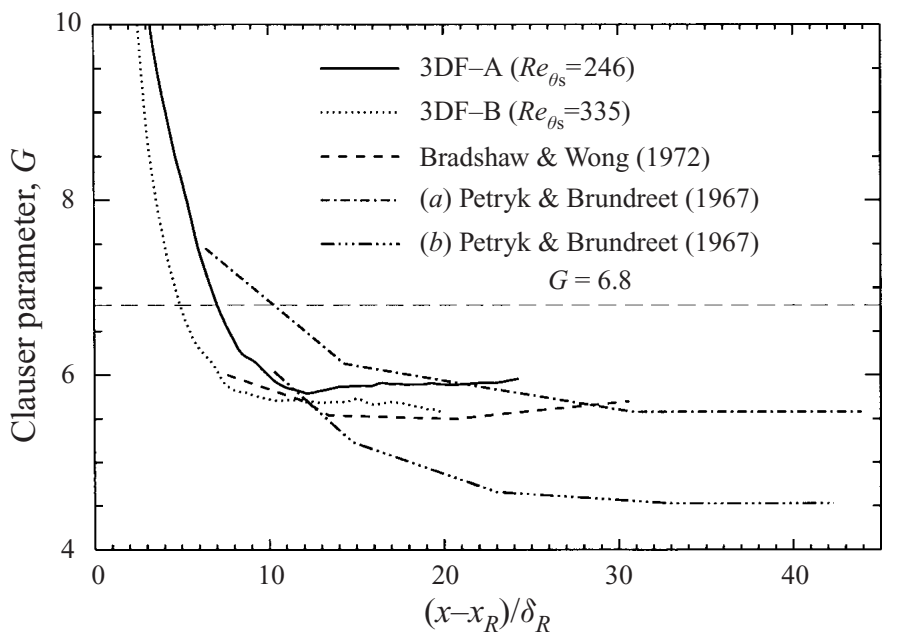

Figure 18. Comparison of Clauser parameter, $\delta_{R} / h=5.8(a), 3.47(b)$.

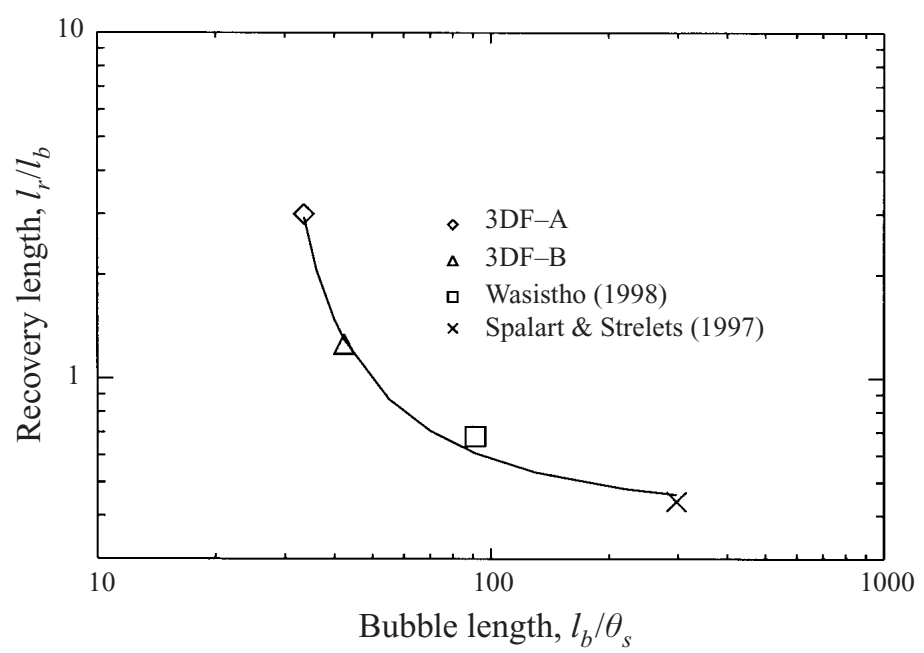

FIGURE 19. Comparison of the non-dimensional bubble length and the recovery length on a log-log plot.

It can be seen that the distance to the skin friction peak relative to bubble length decreases as the ratio of bubble length to momentum thickness at separation increases. This implies that shorter separation bubbles have a proportionately longer initial recovery region.

\section{Stability characteristics of reverse-flow profiles}

The separated shear layer is highly unstable, but a distinction needs to be drawn between convective instability, where disturbances grow in space, and absolute instability where disturbances grow in time. The distinction was first made by Gaster (1963, 1968) and major developments since then are given in Huerre \& Monkewitz (1985, 1990). The distinction may well be important for laminar separation bubbles. Analysis performed by Niew (1993) for backward facing step flows suggests absolute instability 


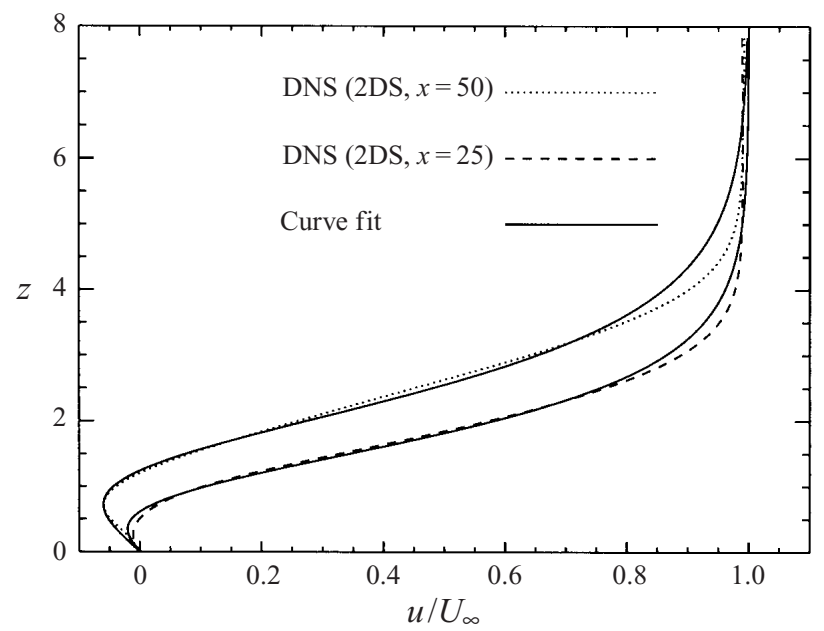

FIGURE 20. Comparison of simulation profiles with an analytic (equation 14) profile. Curve fits at $x=25(A=0.882, B=1.604)$ and at $x=50(A=1.095, B=1.956)$.

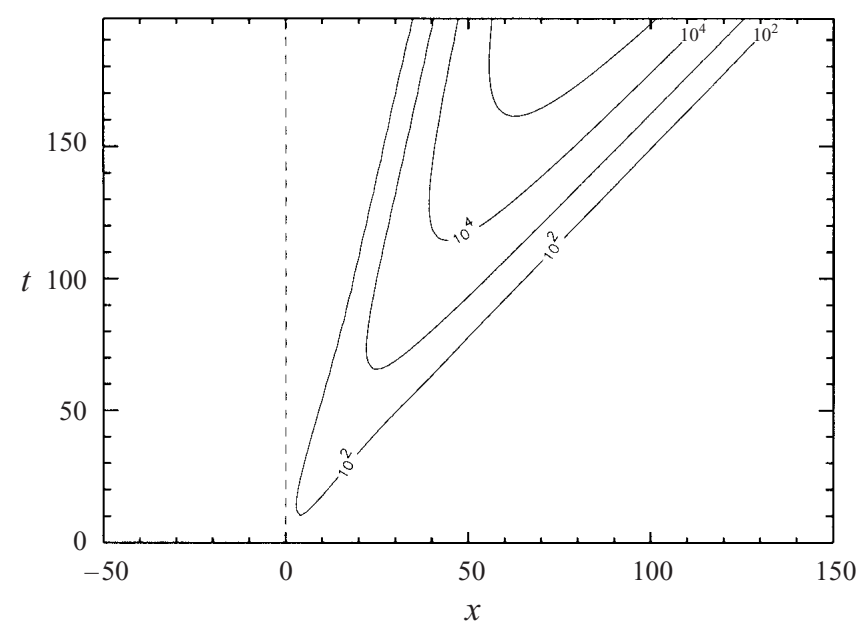

Figure 21. Convectively unstable $5 \%$ reverse flow $(A=0.9, B=1.55)$ profile at $R e_{\delta} *=500$ (contours of $|G|$ ).

for flow with more than $20 \%$ reverse flow, whereas Hammond \& Redecopp (1998) predicted $30 \%$ for the start of absolute instability in laminar separation bubbles. One effect of the presence of a region of local instability would be a global response of the bubble as a whole and this may be the explanation for the large-scale vortex shedding that was observed by Pauley et al. (1990) in two-dimensional simulations for large values of the suction strength. The phenomenon may also be related to the bursting process whereby a short bubble bursts and either forms a long bubble or fails to reattach at all.

We consider here the instability characteristics of velocity profiles that match mean velocity profiles extracted from the simulations. An equation that achieves this is

$$
\frac{u}{U_{\infty}}=\tanh (z)-2 A \frac{\tanh (z / B)}{\cosh ^{2}(z / B)},
$$




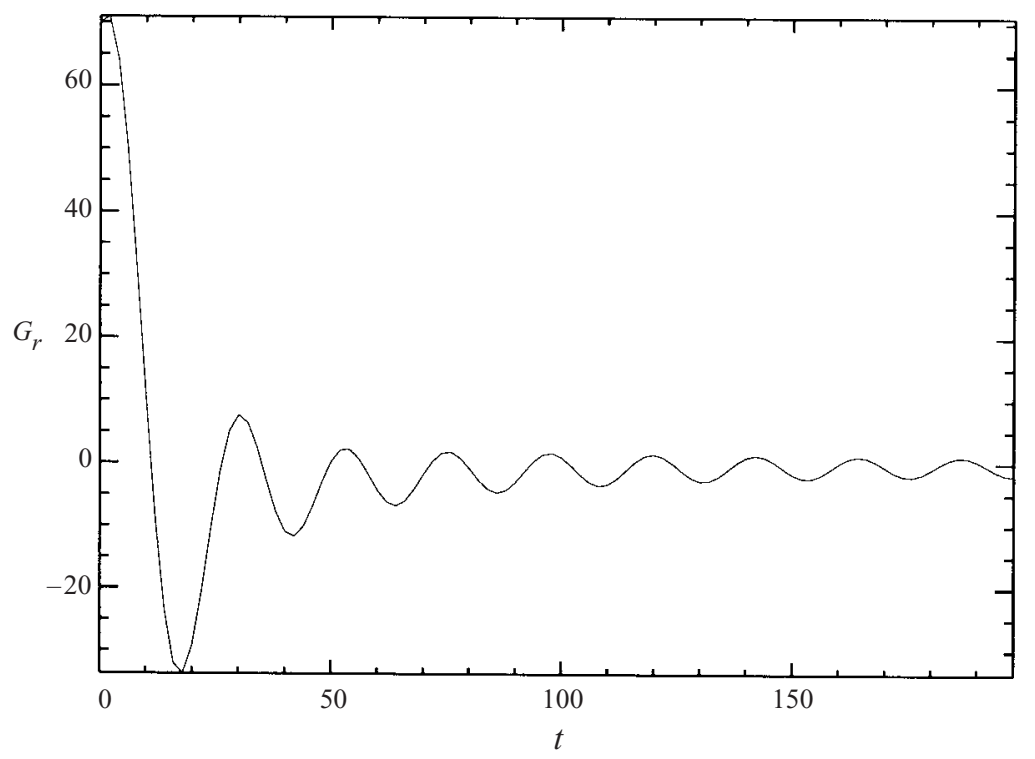

FiguRE 22. Convectively unstable $5 \%$ reverse flow profile at $R e_{\delta}^{*}=500$ $\left(G_{r}\right.$ along the line $\left.x=0\right)$.

where the constants $A$ and $B$ can be adjusted to fit particular profiles from the numerical simulations or used for parametric studies independent of profiles measured in the simulation. Fitting an analytic profile to the data is considered preferable to using the raw data due to the availability of analytic derivatives. A comparison of the fitted profile to a profile from the simulation is shown on figure 20 for two profiles, one in the dead-air region and the other inside the reverse-flow vortex.

To investigate the absolute or convective nature of the instability of profiles given by (14) we employ the method of Niew (1993), who modified Gaster's (1981) wavepacket for an impulse response to provide a simple and cheap method to study the absolute or convective nature of simple plane shear flows. In Niew's method the impulse response of a particular profile is reformulated as a complex summation of the temporal dispersion relation given by

$$
G(x, t)=\sum_{j=1}^{J} \mathrm{e}^{\mathrm{i}\left(\alpha_{j} x-\omega_{j} t\right)}
$$

where the complex frequency $\omega$ for each real value of $\alpha_{j}$ is computed from the Orr-Sommerfeld equation

$$
\left(\bar{U}-\frac{\omega}{\alpha}\right)\left[\hat{u}^{\prime \prime}-\alpha^{2} \hat{u}\right]-\bar{U}^{\prime \prime} \hat{u}=-\frac{\mathrm{i}}{\operatorname{Re} \alpha}\left[\alpha^{4} \hat{u}-2 \alpha^{2} \hat{u}^{\prime \prime}+\hat{u}^{\prime \prime \prime}\right],
$$

where $\bar{U}$ is the velocity profile and derivatives with respect to $z$ are shown by primes. It should be noted that locally parallel flow is assumed in the derivation of (16). The summation in (15) is carried out over the unstable range of waves. Figure 21 shows $G$ on an $x, t$ graph to illustrate the nature of the results for a profile with $A=0.9$ and $B=1.55$, corresponding to a velocity profile with a maximum $5 \%$ reverse flow. It can be seen that the whole wavepacket moves to increasing $x$ as time increases, indicating convective instability. This is confirmed by observing that the variation 


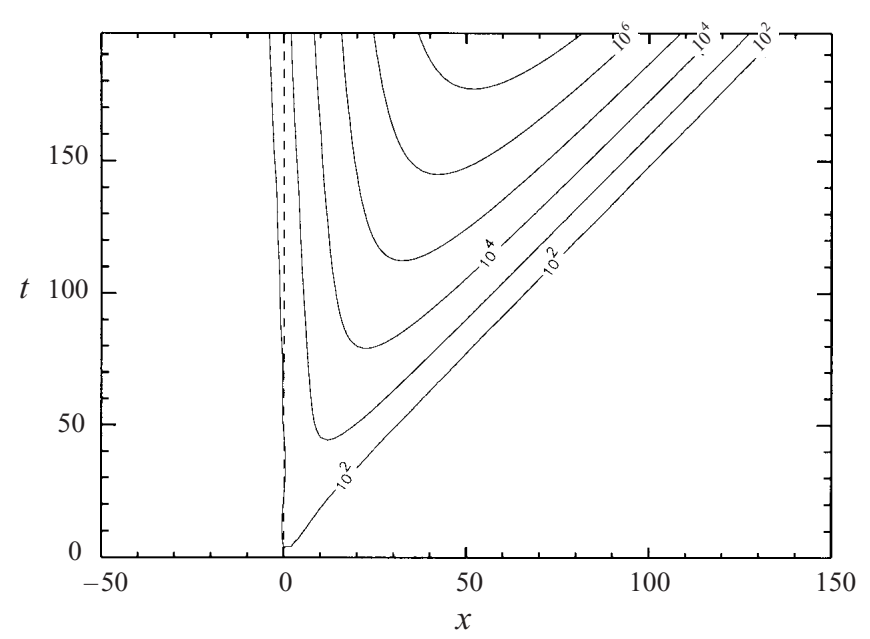

Figure 23. Absolutely unstable $20 \%$ reverse flow $(A=1.16, B=1.55)$ at $R e_{\delta} *=500$ (contours of $|G|$ ).

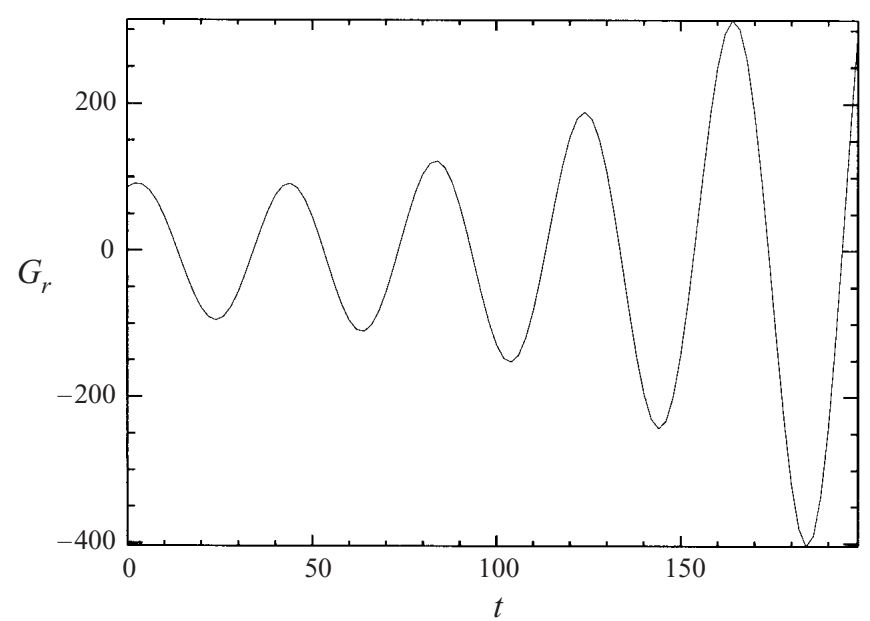

FIGURE 24. Absolutely unstable $20 \%$ reverse flow profile at $R e_{\delta} *=500$ $\left(G_{r}\right.$ along the line $\left.x=0\right)$.

with time of real part $G_{r}$ at $x=0$ is a decaying oscillation (figure 22). By contrast results from a case with $A=1.16, B=1.55$, corresponding to $20 \%$ reverse flow, are shown on figures 23 and 24 . It can be seen that this case is clearly absolutely unstable as the wavepacket crosses the $x=0$ axis. Many such results are summarized on figure 25 on a graph of percentage reverse flow against Reynolds number based on displacement thickness. The solid line divides absolutely unstable profiles from convectively unstable profiles. At large Reynolds numbers the flows are absolutely unstable for reverse flows in excess of $15 \%$, while at the Reynolds numbers typically encountered in separation bubbles the dividing line increases to around $20 \%$. These values may be compared to the maximum mean reverse flow found in the numerical simulation which was $4 \%$ for simulation $3 \mathrm{DF}-\mathrm{A}$ and $8 \%$ for $3 \mathrm{DF}-\mathrm{B}$. We also note from the probability density functions of $\S 2.4 .2$ (see figure 10) that instantaneous reverse flows of more than $15 \%$ were only found less than $1 \%$ of the time. Thus 


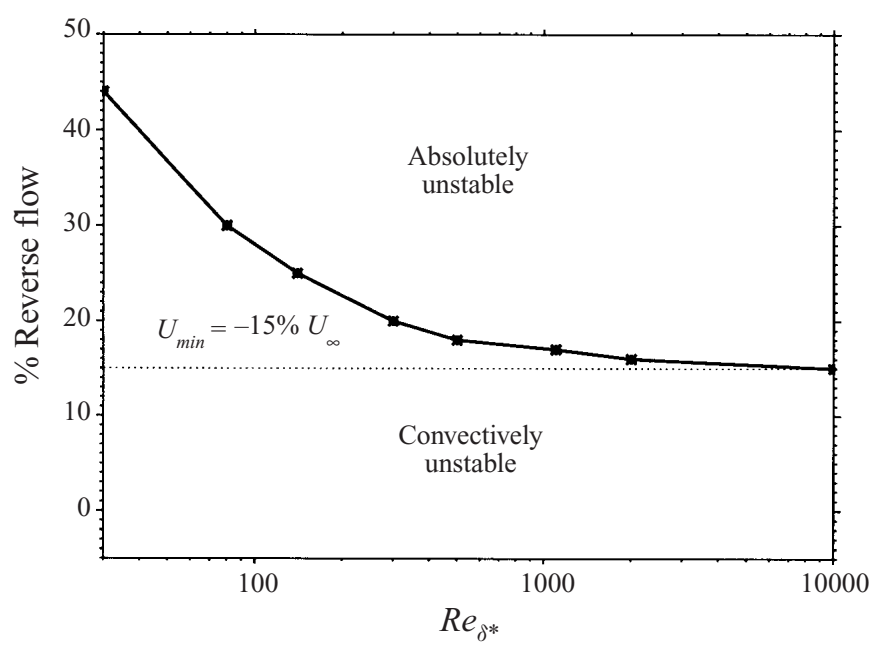

FIGURE 25. Margin of absolute/convective stability.

all the indications are that the bubbles simulated here are properly classified as convectively unstable. Allen \& Riley (1995) reached a similar conclusion for a set of separation bubbles defined by interacting boundary-layer theory and an algebraic eddy-viscosity turbulence model.

We have seen that increases in the amount of reverse flow may lead to absolute instability. Factors which affect the reverse flow include strength of the adverse pressure gradient and Reynolds number. An example of a laminar separation bubble with much larger amounts of reverse flow is that of Spalart \& Strelets (1997), who observed a maximum $23 \%$ reverse flow. Since their bubble would be classed as 'long' (a factor of 10 longer than 3DF-A when the bubbles are normalized with momentum thickness at separation) the question arises as to whether absolute instability is a characteristic of long bubbles and convective instability a characteristic of short bubbles. If that were true then a movement of stability characteristics from convective towards absolute would be indicative of an imminent burst of a short bubble to a long bubble. More simulations, and specifically simulations of bubbles undergoing bursting, are required to clarify this issue. An alternative explanation was offered by Gaster (1969), who postulated a global instability of the bubble and the external potential flow as being responsible for bursting, rather than some local property of the bubble itself.

\section{Comparison of two-dimensional and three-dimensional simulations}

Two-dimensional simulations are cheap to run, and it is important to know which properties of simulation bubbles can be predicted with such methods and which cannot. Ripley \& Pauley (1993) compared results from two-dimensional simulations with Gaster's $(1963,1969)$ experiments and found good agreement in terms of separation and reattachment points, and pressure plateau in the upstream portion of the bubble. Maucher, Rist \& Wagner (1994) also carried out two-dimensional simulations but stressed the need for fully three-dimensional simulations to capture some basic features of separated or strongly decelerated flow.

Before comparing results of two-dimensional and three-dimensional simulations the issue of self-sustained oscillations and vortex shedding needs to be discussed. 

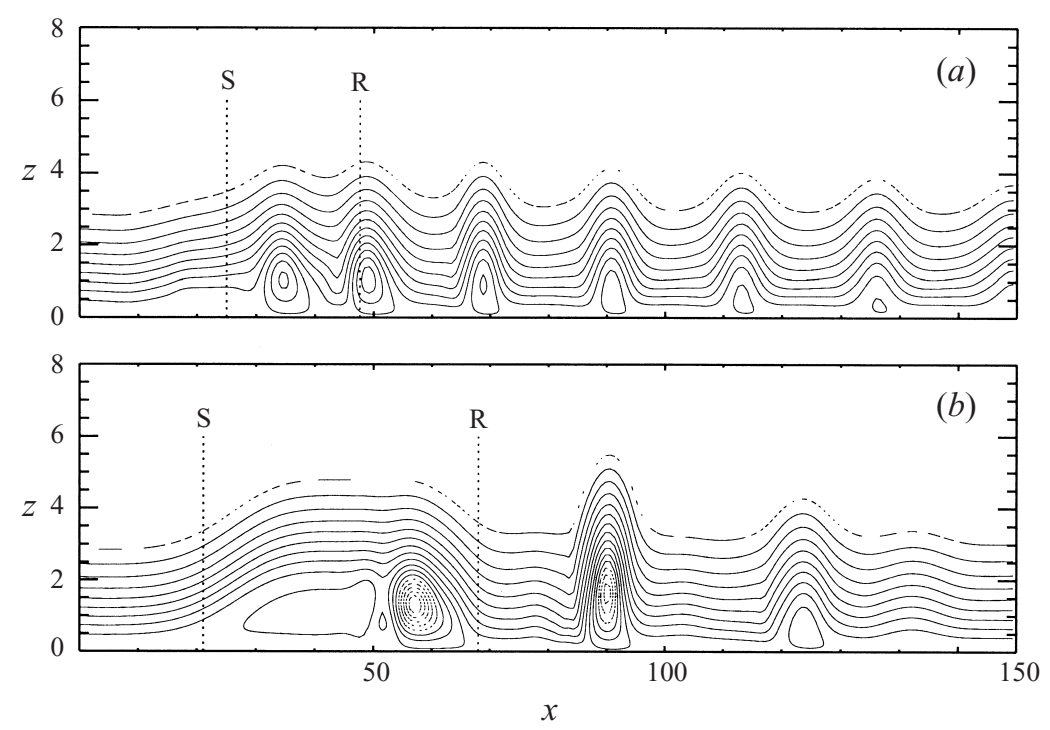

Figure 26. Contours of stream function. (a) 2DF and (b) 2DS. Contour levels have been selectively chosen to show the presence of the vortex structures.

Pauley et al. (1990) carried out simulations for a range of suction strengths and found an irregular vortex shedding for large values of suction strength. The shedding persisted even without external forcing of the flow. A similar phenomenon has been observed by all subsequent researchers, though there is disagreement about a precise shedding criterion (Wasistho 1998). Alam \& Sandham (1997) also found self-sustained shedding but reported that this was due to the effects of the buffer zone in the numerical simulation method and not necessarily a physical phenomenon. The buffer zone does not damp disturbances completely. Those that pass through the domain can interact with the flow, effectively setting up a feedback loop, which manifests itself as vortex shedding. No numerical boundary scheme is completely free from reflections which can set up global responses (see also Buell \& Huerre 1988) and it appears that a conclusive study of the phenomenon is not possible at present. Certainly a much more careful study of inflow and outflow boundary conditions is required. Of the two-dimensional simulations given in tables 1 and 2, one (2DF) is for a forced two-dimensional bubble, while the other (2DS) is for a bubble which exhibits self-sustained vortex shedding.

To illustrate the differences between two-dimensional and three-dimensional simulations we make comparisons between two-dimensional and three-dimensional simulations, whose numerical parameters were shown on tables 1 and 2 and main flow properties on table 3. Instantaneous views of the two-dimensional simulations are shown on figure 26. The most direct comparison is between simulations $2 \mathrm{DF}$ and 3DF-A which are set up to be the same except for the forcing, which has a sinusoidal spanwise variation for the three-dimensional case. Skin friction is shown on figure 27 for comparison with figure 6 . It can be seen that the bubble for the $2 \mathrm{DF}$ calculation is $40 \%$ longer than the corresponding three-dimensional calculation 3DF-A, while the minimum skin friction is only half that of the three-dimensional simulation. The behaviour of the skin friction and also turbulence properties (not shown) after reattachment is obviously different since near-wall turbulence cannot be remotely approximated by two-dimensional simulation. 


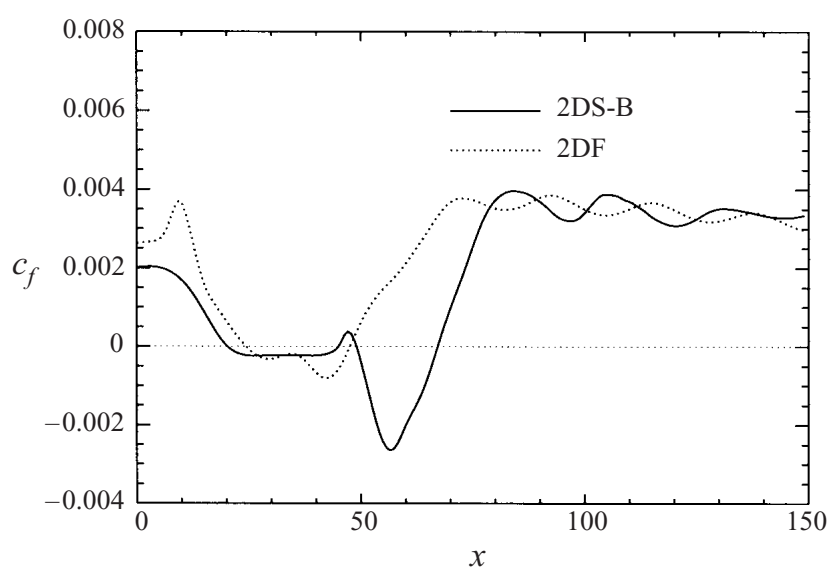

FIGURE 27. Skin friction variation of the two-dimensional bubbles.

The good agreement with experiments found by Ripley \& Pauley (1993) for bubble length may have been due to specification of the exact experimental pressure distribution as the upper boundary condition for the simulations. From the present results one would expect that bubbles with three-dimensional breakdown to turbulence are fundamentally different to two-dimensional bubbles. An exception to this may be three-dimensional simulations of bubbles at high suction strengths that may exhibit vortex shedding. The simulation with high reverse flows by Spalart \& Strelets (1997) did find shear-layer flapping, but no comparable two-dimensional simulation was available.

\section{Conclusions}

Simulations of short laminar separation bubbles have been carried out in two and three dimensions. The three-dimensional simulations show full transition to turbulence, the process being characterized by breakdown of $\Lambda$-vortices. Budgets of turbulence kinetic energy and Reynolds stresses show that the flow just after reattachment consists of an upper region, corresponding quite closely to the upper half of a turbulent mixing layer and a near-wall portion with a redeveloping turbulent boundary layer. The relaxation towards equilibrium is slow. At least seven bubble lengths are required to reach the usual log law. Stability analysis showed that profiles with more than $15 \%$ reverse flow were required for absolute instability, whereas the bubbles simulated had lower levels of reverse flow. Two-dimensional simulations do not appear to represent adequately the characteristics of the short separation bubbles. Further simulations should address the issue of bursting of short separation bubbles to form long bubbles.

The authors would like to thank British Aerospace, Sowerby Research Centre for their financial support of this work and Dr H. P. Horton and Professor M. Gaster for helpful suggestions during the research. National supercomputer time was provided by EPSRC under grants GR/K43957 and GR/M08424.

\section{REFERENCES}

Alam, M. 1998 Direct numerical simulation of laminar separation bubbles. PhD Dissertation, Queen Mary and Westfield College, University of London. 
Alam, M. \& Sandham, N. D. 1997 Simulation of laminar separation bubble instabilities. In Direct and Large Eddy Simulation II (ed. J. P. Chollet et al.), pp. 125-136. Kluwer.

Alam, M. \& Sandham, N. D. 1998 Numerical study of separation bubbles with turbulent reattachment followed by a boundary layer relaxation. In Parallel Computational Fluid Dynamics (ed. D. R. Emerson et al.), pp. 571-578. Elsevier.

Allen, T. \& Riley, N. 1995 Absolute and convective instabilities in separation bubbles. Aeronaut. J. 99, 439-448.

Bradshaw, P. \& Wong, F. Y. F. 1972 The reattachment and relaxation of a turbulent shear layer. J. Fluid Mech. 52, 113-135.

Buell, J. C. \& Huerre, P. 1988 Onflow/outflow boundary conditions and global dynamics of spatial mixing layers. Rep. CTR-S88, pp. 19-27. Center for Turbulence Research, Stanford University.

Canuto, C., Hussani, M. Y., Quarteroni, A. \& Zang, T. 1988 Spectral Methods in Fluid Dynamics. Springer.

CAstro, I. P. \& EpIK, E. 1996 Boundary layer relaxation after a separated region. Expl Thermal Fluid Sci. 13, 338-348.

Davis, R. L., Carter, J. E. \& Reshotko, E. 1985 Analysis of transitional separation bubbles on infinite swept wings. AIAA Paper 85-1685.

Gaster, M. 1963 On stability of parallel flows and the behaviour of separation bubbles. PhD Dissertation, University of London.

Gaster, M. 1968 Growth of disturbances in both space and time. Phys. Fluids 11, 723-727.

GASTER, M. 1969 Structure and behaviour of laminar separation bubbles. ARC R\&M 3595. HMSO.

Gaster, M. 1981 Propagation of linear wave packets in laminar boundary layers. AIAA J. 19, $419-423$

Guo, Y., Kleiser, L. \& AdAms, N. A. 1994 A comparison study of an improved temporal DNS and spatial DNS of compressible boundary layer transition. AIAA Paper 94-2371.

Hammond, D. A. \& RedeKopp, L. G. 1998 Local and global instability properties of separation bubbles. Eur. J. Mech. B/Fluids 17, 145-164.

HILDINGS, C. 1997 Simulations of laminar and transitional separation bubbles. TR S-100 44. Royal Inst. of Tech., Stockholm, Sweden.

Horton, H. P. 1967 A semi-empirical theory for the growth and bursting of laminar separation bubbles. ARC Conf. Proc. 1073.

Horton, H. P. 1968 Laminar separation in two and three-dimensional incompressible flow. PhD Dissertation, University of London.

Howard, R. J. A. \& Sandham, N. D. 1997 Simulation and modelling of the skew response of turbulent channel flow to spanwise flow deformation. In Direct and Large Eddy Simulation II (ed. J. P. Chollet et al.), pp. 125-136. Kluwer.

Huerre, P. \& Monkewitz, P. A. 1985 Absolute and convective instabilities in free shear layers. J. Fluid Mech. 159, 151-168.

Huerre, P. \& Monkewitz, P. A. 1990 Local and global instabilities in spatially developing flows. Ann. Rev. Fluid Mech. 22, 473-537.

Jones, B. M. 1938 Stalling. J. R. Aero. Soc. 38, 747-770.

Kim, J., Moin, P. \& Moser, R. 1987 Turbulent statistics in fully developed channel flow at low Reynolds number. J. Fluid Mech. 177, 133-166.

Kleiser, L. \& SCHUMANN, U. 1980 Treatment of incompressibility and boundary layer conditions in three-dimensional numerical spectral simulations of plane channel flows. In Proc. 3rd GAMM Conf. on Numerical Methods in Fluid Mechanics (ed. Hirschel, E. H.), pp. 165-173. Vieweg.

LE, H., MoIn, P. \& KIM, J. 1997 Direct numerical simulation of turbulent flow over a backward-facing step. J. Fluid Mech. 330, 349-374.

Lin, J. C. M. \& Pauley, L. L. 1996 Low-Reynolds-number separation on an aerofoil. AIAA J. 34, $1570-1577$.

Maucher, U., Rist, U. \& Wagner, S. 1994 Direct numerical simulation of aerofoil separation bubbles. ECCOMAS 94. Wiley \& Sons.

NiEw, T. R. 1993 The stability of the flow in a laminar separation bubble. PhD Dissertation, University of Cambridge.

Owen, P. R. \& KLANFER, L. 1953 On the laminar boundary layer separation from the leading edge of a thin aerofoil. ARC Conf. Proc. 220. 
Pauley, L. L. 1994 Response of two-dimensional separation to three-dimensional disturbances. Trans. ASME I: J. Fluids Engng 116, 433-438.

Pauley, L. L., Moin, P. \& Reynolds, W. C. 1990 The structure of two-dimensional separation. J. Fluid Mech. 220, 397-411.

Petryk, S. \& Brundreet, E. 1967 Res. Rep. 4. Dept. of Mech. Engng, University of Waterloo.

Ripley, M. D. \& Pauley, L. L. 1993 The unsteady structure of two-dimensional separation. Phys. Fluids A 5, 3099-3106.

Rist, U. 1994 Nonlinear effects of two-dimensional and three-dimensional disturbances on laminar separation bubbles. IUTAM Symp. on Nonlinear Instability of Nonparallel Flows, NY, USA (ed. S. P. Lin). Springer.

RoBerTs, W. B. 1980 Calculation of laminar separation bubbles and their effect on aerofoil performance. AIAA J. 18, 25-31.

Robinson, S. K. 1991 The kinematics of turbulent boundary layer structure. NASA TM 103859.

Rogers, M. M. \& Moser, R. D. 1994 Direct simulation of a self-similar mixing layer. Phys. Fluids 6, 903-923.

SAndham, N. D. \& Howard, R. J. A. 1998 Direct simulation of turbulence using massively parallel computers. In Parallel Computational Fluid Dynamics (ed. D. R. Emerson et al.), pp. 23-32. Elsevier.

Sandham, N. D. \& Kleiser, L. 1992 The late stages of transition to turbulence in channel flow. J. Fluid Mech. 245, 319-348.

SpalaRt, P. 1988 Direct simulation of a turbulent boundary layer up to $R e_{\theta}=1410$. J. Fluid Mech. 187, 61-98.

Spalart, P. R. \& Strelets, M. K. 1997 Direct and Reynolds-averaged numerical simulation of a transitional separation bubble. 11th Symp. on Turbulent Shear Flows, Grenoble, France.

Spalart, P. R. \& WatmufF, J. H. 1993 Experimental and numerical study of a turbulent boundary layer with pressure gradients. J. Fluid Mech. 249, 337-371.

TheOdORSON, T. 1955 The structure of turbulence. In 50 Jahre Grenzschichtforchung (ed. H. Görtler \& W. Tollmien). Vieweg.

WASistho, B. 1998 Spatial direct numerical simulation of compressible boundary layer flow. Thesis, Universiteit Twente, Enschede, The Netherlands.

Wilson, P. G. \& Pauley, L. L. 1995 Two-dimensional large eddy simulation of a transitional separation bubble. Symp. on Separated and Complex Flows, ASME/JSME Fluid Engineering Conf.

Young, A. D. \& Horton, H. P. 1966 Some results of investigation of separation bubbles. AGARD CP, No. 4, pp. $779-811$. 